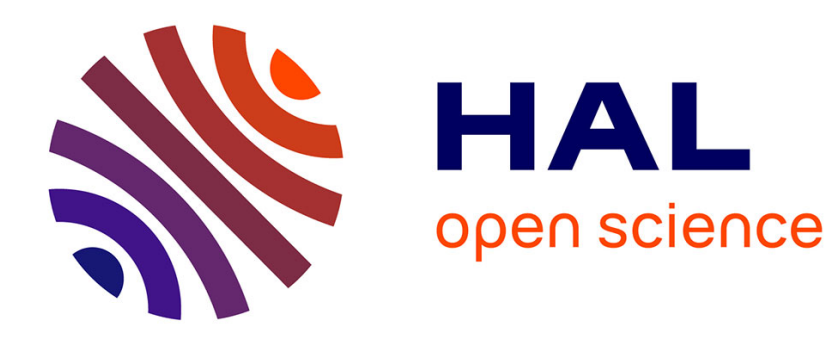

\title{
de natura animae psychologiae principia mathematica et philosophyae naturalis
}

\author{
José Luís Simões da Fonseca
}

\section{To cite this version:}

José Luís Simões da Fonseca. de natura animae psychologiae principia mathematica et philosophyae naturalis. Gatha Cognition, 2017, 978-81-932622-1-4. 10.21523/gcb3 . hal-03302619

\section{HAL Id: hal-03302619 \\ https://hal.science/hal-03302619}

Submitted on 27 Jul 2021

HAL is a multi-disciplinary open access archive for the deposit and dissemination of scientific research documents, whether they are published or not. The documents may come from teaching and research institutions in France or abroad, or from public or private research centers.
L'archive ouverte pluridisciplinaire HAL, est destinée au dépôt et à la diffusion de documents scientifiques de niveau recherche, publiés ou non, émanant des établissements d'enseignement et de recherche français ou étrangers, des laboratoires publics ou privés. 


\section{de natura animae psychologiae principia mathematica et philosophyae naturalis}

\section{José Luís Simões da Fonseca}

Chairman of Psychology and Psychiatry, Faculty of Medicine of the University of Lisbon (Emeritus) Director, Department of Psychiatry of Hospital de Santa Maria, Lisbon (Emeritus)

Coordinator of the Area of Mental Health Psychology and Psychiatry, Faculty of Medicine of Lisbon (Emeritus)

Active member of the Center for Philosophy of Science of the University of Lisbon (CFCUL), Lisbon

Center for Philosophy of Science of the University of Lisbon (CFCUL)

Faculty of Science, University of Lisbon, Lisbon 


(C) J.L. Simões da Fonseca, 2015

Title: de natura animae. psychologiae principia mathematica et philosophyae naturalis

Author: José Luís Simões da Fonseca 




\section{de natura animae}

\section{psychologiae principia mathematica et philosophyae naturalis}

\section{José Luís Simões da Fonseca}

Chairman of Psychology and Psychiatry, Faculty of Medicine of the University of Lisbon (Emeritus) Director, Department of Psychiatry of Hospital de Santa Maria, Lisbon (Emeritus)

Coordinator of the Area of Mental Health Psychology and Psychiatry, Faculty of Medicine of Lisbon (Emeritus)

Active member of the Center for Philosophy of Science of the University of Lisbon (CFCUL), Lisbon

Center for Philosophy of Science of the University of Lisbon (CFCUL)

Faculty of Science, University of Lisbon, Lisboa 

To Warren Sturgis McCulloch, In Memoriam 

"When Magellan sailed along the American shore with the intention of finding a western passage, he had no inductive evidence that there was one; but his enterprise was justified because it was a means to reach his aim if the aim was attainable."

Hans Reichenbach 



\section{Index}

"How is it that sensing so much we can do so little?

How is it that sensing so little we can do so much?"

Karl H. Pribram

Index 1

Preface 3

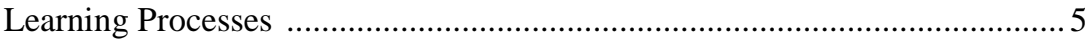

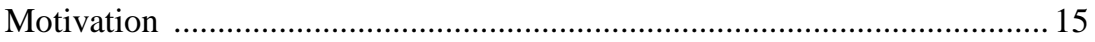

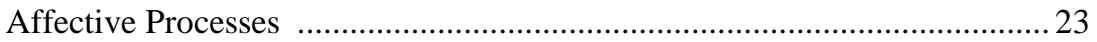

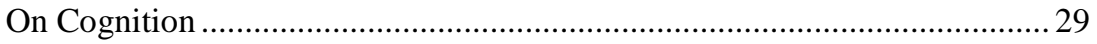

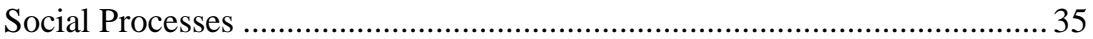

The Hodgkin Huxley study of action potential ............................................. 39

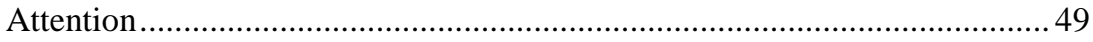

Memory and the problem of automata reproduction ...................................51

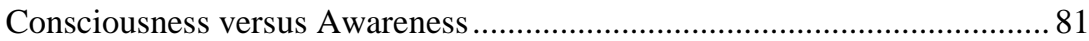

Herbert Spencer and Hughlings proposal for neural central nervous system

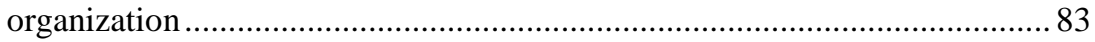

Genetic mutations and auto and self-organization..................................... 87

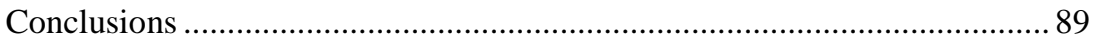

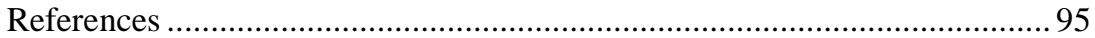

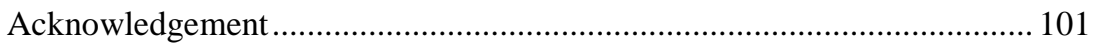





\section{Preface}

A first question that needs to be clarified is if there are arguments enough to assert or deny a Quantum character in the morphology and physiology of the Central Nervous System.

The genial Spanish neuroanatomist Santiago Ramón y Cajal (1934) demonstrated after a long controversy, that neurocells are discrete elements separated from each other, and from glial cells. Lord Adrian (1922) as well as Bowditch (1871) recorded action potentials which obeyed the all or nothing law, and the former was awarded a Nobel Prize for it. There are, nevertheless, parts and sub-systems of the CNS that morphologically are nor discrete neither modular, and do not function in a quantum manner, for instance the molecular biology mechanisms of learning and metabolism.

In psychology we are confronted with a singular question: concepts that are used in a scientific or in a professional praxis are still being introduced, and it's even discussed if a psychology in the third person is legitimate when we lack a psychology in the first person, as this problem is commented by phenomenologists like Sass, Parnas (2011) and Zahavi (Zahavi, 2005) in Denmark.

Therefore, we have to undergo a process of concept selection, construct establishment and double symmetric validation of both psychological and physiological constructs.

In our endeavor, we first tried, under the influence of Warren McCulloch to write what we called a Tractatus Logico-Psicologicus. The present essay was stimulated by Professor Filmer Northrop of the University of Yale, New Haven, Connecticut, that believed in (and even told Heisenberg about) the existence of our project. 



\section{Learning Processes ${ }^{1}$}

Due to its apparent simplicity, learning processes have been adopted during one hundred years as the domain in which the most successful theoretical constructions have been obtained in psychology. A dog of Pavlov in his memories wrote: "I had a master so intelligent that only through the pressing of a lever I was able to make him give me food". Objectively, the science of psychology, as a science of public evidence of behavior, was largely based on the analysis of the behavior of cats, dogs, pigeons and so forth, being doubtful if all those animal models were adequate enough. As a matter of fact, they were at a very far distance from normal, usual behavior models studied by ethologists, like for instance Tinbergen, Conrad Lorenz, Don Holst. The main point these authors made was that the behaviors they studied were natural with animals, in certain and common situations of their natural life.

As a matter of belief, ethologists had the persuasion that behavior is much more a matter of inheritance than of acquisition of new experience. Anyway, no great synthesis has been born after the great investments in investigation from a point of view that has take into account natural conditions. The Naked Ape of Desmond Morris (1967) is an example of how these metaphors are not sufficient cogent to catch the preference of scientists. Historically, the Russian investigators, Pavlov and Sechenov establish the laws of association, reinforcement, extinction, and arousal. They did not apply these laws of conditioning to behaviors that involved language. And that rendered impossible the study with their method of cognition. In the traditional associative approach, it was studied the association between conditional and

1 With the colaboration of I. Barahona da Fonseca, Faculty of Psychology of the University of Lisbon and J. Barahona da Fonseca, Faculty of Sciences and Technology, New University of Lisbon 
non-conditional stimuli but alternative strategies which involve association and stimuli and responses were theoretically used to build models of learning. One important way of understanding these behaviors was to relate them with the results that animals obtained, by using them. That is, for instance, the degree of reinforcement by satisfaction of their needs. The law of precedence of unconscious stimuli by condition stimuli, or the conditions for the mass conditioning were studied, besides the involvement of the cortex that had been proposed by Pavlov, Sechenov and others. It began a study involving amygdala, hippocampus, the cerebellum, the reticular system of the brain stem and so forth. This interminable study is not in the core of our inquiry. We are more interested in invariants than in detailed alternatives.

\section{The Hull Paradigm}

Hull (1943) proposed a famous law for determining the probability of a behavior by the expression:

$$
P(s, r, m)=D(m) H(s, r)
$$

In which $H$, the habit, means the strength for the association between stimulus $s$ and responses $r$ and $D$, the value of the drive depending on the intensity of the motivation $m$.

Considering the occurrence of two simultaneous processes of learning we may write

$$
P^{\prime}\left(\mathrm{s}, \mathrm{r}, \mathrm{s}^{\prime}, \mathrm{r}^{\prime}\right)=D_{m} H(s, r) \cdot\left(1-H^{\prime}\left(s^{\prime}, r^{\prime}\right)\right)
$$

Where the complement of the probability means that the second 
process of learning is considered not to occur together with the first process that has for probability $(s, r, m)$.

Considering the short period of time in which learning takes place, we may, for simplicity reasons, assume that $D$ has a constant value $k$.

Let us now assume that learning implies an increase of the strength of the habit $H$. On the other hand, we have to take into account the value the static value $H$.

The equation describing the overall process may be written

$$
A_{1} \frac{\partial^{2} H}{\partial s^{2}}+A_{2} \frac{\partial^{2} H}{\partial r^{2}}+A_{3} \frac{\partial H}{\partial s}+A_{4} \frac{\partial H}{\partial r}+A_{5} H=P
$$

As in general the nervous system is never totally involved in a single process, but rather only a part of it, we express this fact introducing the variable $\mathrm{c}$ (commitment) which is a dimensional variable, which partitions the global process, defining an effectively operant part of it.

This may be written as

$$
P / c=c \cdot H(s, r)
$$

Taking into account the variation in probability due both to stimuli and responses, as $s$ and $r$ are functions of $t$, implicitly a tax or variation of $H(s, r)$ can be defined relatively to time.

The commitment of neurons in $H$ becomes:

$$
\frac{P(s, r)}{n}=c
$$


In which $n$ is the total number of Central Nervous System (CNS) units involved in the process. Considering the system submitted to an oscillatory period,

$$
2 \pi \omega \cdot P(s, r)=B_{1} \frac{\partial^{2} H}{\partial t^{2}}+B_{2} \frac{\partial H}{\partial t}+B_{3} H(s, r)
$$

Relationships between two stimuli $s_{1}, s_{2}$, two responses $r_{1}, r_{2}$, have to be taken into account, as well as the relationships between responses and stimuli. The full combinatory becomes: $H\left(s_{1}, r_{1}\right)$ and $H\left(s_{2}, r_{2}\right)$.

If we consider what happens during the process of learning it is necessary to notice that the total equation expresses a probability and therefore it must be guaranteed that the result is included in a closed interval:

$$
\begin{gathered}
P_{1}=A_{1} \frac{\partial^{2} H}{\partial s_{1}}+A_{2} \frac{\partial^{2} H}{\partial r_{1}}+A_{3} \frac{\partial H}{\partial s_{1}}+A_{4} \frac{\partial H}{\partial r_{1}}+A_{5} H\left(s_{1}, r_{1}\right) \\
P_{2}=A_{1} \frac{\partial^{2} H}{\partial s_{2}}+A_{2} \frac{\partial^{2} H}{\partial r_{2}}+A_{3} \frac{\partial H}{\partial s_{2}}+A_{4} \frac{\partial H}{\partial r_{2}}+A_{5} H\left(s_{2}, r_{2}\right) \\
0<P_{1} . P_{2} \leq 1
\end{gathered}
$$

We have here a double process of learning: in the first part of the process, it occurs an acquisition of a response, whose probability will increase from near 0 to 1 ; In the second part of the process we have a process with arbitrary probability $P_{2}$, which will suffer extinction, which implies that $P_{2}$ will approach 0 , remaining in its vicinity.

If both the dynamic and the static parts of the formula isolated or together exceed 1 , then an absorbing barrier will make it equal to 1 , and if the same parts of the equations tend to 0, the barrier will avoid it, if exceeds it in 
negative direction. Probability 1 is linked to the end of the acquisition of the learning process and probability 0 to the end of extinction process. From an informational point of view, in the strict sense, we cannot apply mathematical theory of communication due to the stochastic process: what we are talking about is not stationary and natural, it isn't free of learning. Therefore, it is not licit to use it in the strict sense.

Nevertheless, if we consider a small enough interval, we may take these values as constant for the interval and then apply the mathematical treatment proposed by Shanon and Weaver (1949) for measuring entropy in the mathematical theory of communication. In this context, probability 1 is linked to entropy 0 and probability 0 is linked to probability $\infty$. The intermediate values are much more difficult to calculate. In what concerns constants for the dynamic part of the equation, it is reasonable to admit that they are point 1 or point 2 according to the argument we will develop next. For $H(s, r)$, the coefficient should be 1 for the reasons we will next discuss. Still, the dynamic and the static part of the equation will remain always between 0 and 1 . How do we calculate the coefficients for the dynamic part of the equation? What has more weight? The second derivative or the first derivative, that is, the speed of learning?

In animal learning the process of acquisition is more steadily slow than in human beings. As a matter of fact, humans use cognition as much as learning in the strict sense. Cognition is based in positing hypothesis and in the process of insight. Our group made an experiment in 1978 in which we supposed that each state of the system, during the process of learning, resulted in a transformation of the proceeding state.

$$
\begin{aligned}
& t . s(t-1) \equiv s(t) \\
& y(t)+1 \equiv s(t)
\end{aligned}
$$


In a practical description, subjects were submitted to a process in which they should guess which was the decision function more convenient to stop a process of negative reinforcement. They were submitted to completely irrelevant visual stimuli, and to a visual stimuli that would always be followed by a negative event, five seconds after the end of the stimuli, if the subject was not able to propose a correct solution. The subjects had in front of them a button and two keys, linearly placed from left to right. Subjects had the following hypothesis:

(1) Doing nothing (initial inertia);

$$
\bar{D} \bar{E} R
$$

That is, no attempt to solve the problem initially;

(2) Ensaying, pressing the key on the left, followed by negative reinforcement;

$$
\bar{D} E R
$$

(3) Making a correct hypothesis but performing the corresponding behavior more than five seconds after the end of the stimuli, that is, being only able to interrupt negative reinforcements.

$$
\bar{D} \bar{E} R \bigvee D E R
$$

And finally: 
(4) Making the right guess and executing the corresponding behavior less than five seconds after the end of visual stimuli.

\section{$D \bar{E} \bar{R} D E \bar{R}$}

We verified that most of the time the behavior was constituted by pseudostability states in which the same answer was executed during a certain number of epochs. The walk in the space of phases was mainly constituted by this kind of pseudo stabilities or else of short transients almost closing an oscillatory period. A remarkable characteristic was that subjects were not learning in a steady way but rather discontinuously, making jumps in an irregular way, what corresponds in our previous equations to a higher derivative in the dynamic part of the equation. The following graphic helps to understand:

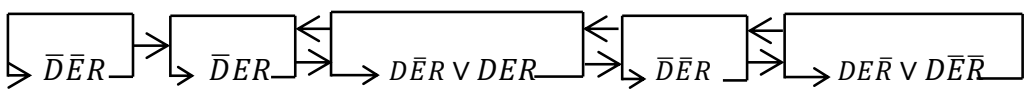

Fig. 1 Initial inactivity; Pressing the key on the left; Interruption of negative reinforcement; Post-initial inertia; Acquisition performed

We are now in the condition to write the eigenfunction and to determine the eigenvalues of the $\Psi$ function of a system. As a matter of fact, what we want to say is that to each kind of organization of a learning system it corresponds a level of energetic and informatics involvement of the components of the system.

Still, there are further issues concerning the characteristics of the system. The theoretical development about structures involved in the understanding of learning made it necessary to take into account the effect of reinforcement on 
learning structures. This key concept of reinforcement makes learning theory merge into theory of motivation.

We learn because the new habits we acquire contribute to satisfy our needs. The level of our motivation for a given behavior is progressively reduced as our needs reduce, or even before this occurs.

This is a form of utilitarian, or so to speak, economic theory of learning.

Nevertheless, present day theory is causal and based in chemical molecular principles.

\section{The present day image of internal representation}

Kandel et al. (2013) and collaborators studied the water snail, aplysia, and co-related learning processes with phenomena observed in the second system of neurotransmission.

Adenilciclase is activated by serotonin, and transforms Adenosinotriphosphate into cyclic adenosine monophosphate (cAMP).

In turn cAMP liberates the catalytic part of protein kinases from it's regulatory component.

Protein kinases then activate great phosphoproteins like ion channels and other macro-structures, phosphorylating them and changing their stereometric properties.

During learning processes, protein kinases accumulate and enhance reaction activities, remaining in the place of the activity. These learning processes and long duration memory are similar in other realms of the animal kingdom, for instance for the terrestrial vertebrates..

In what concerns long duration memory, the brain encodes this information due to the action of hippocampus, which changes short-term memory into long-lasting memory. In cases in which hippocampus has been destroyed in both sides of the brain, this passage from a memory of short duration into a 
memory of long duration does not occur and long experienced situations are repeatedly provoked. A similar phenomenon occurs in Korsakoff syndrome, in which the walls of the ventricle are progressively destroyed due to growing deterioration in neurons due to alcohol abuse.

Naturally long term memory is accessible to experimentation only in animal models. The importance of RNA for memory was noted by Hyden but only after the studies of Kandel et al. (2013) was a coherent conceptualization of the molecular biology processes in long-term memory has been formed.

Protein kinases penetrate the promoter segment of genes and modify gene expression and gene transcription, changing RNA messenger. This may result from pure learning, observed, for instance, during psychiatric treatment. Here pure biology converges with genetics and learning processes. A further advance is that protein kinases suffer a genetic modification improving their efficiency.

The process of encoding itself of this type of actions is performed by protein transcriptase.

This is a single line of approximation to the complex bio-molecular system. There exist systems for bio cellular oxidation and other essential biological processes.

Under normal circumstances, the release of acid CREB-2 which exerts its repressive action and the simultaneous activation of CREB-1 induce the expression of genes, placed subsequently in the causal chain. Two enhanced expressions are particularly important (1) the enzyme ubiquitin carboxyterminal hydrolase, which activates proteasomes to make PKA persistently active, and (2) the transcription factor C/EBP, one of the components of a gene cascade necessary for the growth of new synaptic connections. As it is said by Kandel et al (2013): 
"The induction of the hydrolase is a key step in the recruitment of a regulated proteolytic complex: the ubiquitindependent proteasome. As in other cellular contexts, ubiquitinmediated proteolysis also produces a cellular change of state, here by removing inhibitory constrains on memory. One of the substrates of this proteolytic process is the regulatory subunit of PKA.

PKA is made up of four subunits: two regulatory submits inhibit two catalytic subunits. Long-term training and the induction of the hydrolase degrades about $25 \%$ of the regulatory (inhibitory) subunits in the sensory neurons. As a result, the catalytic subunits continue phosphorylating proteins important for enhance transmitter release and strengthening the synaptic connections, including CREB-1, long after the second messenger, cAMP, has returned to its basal level. This is the simplest mechanism for long-term memory: a secondmessenger kinase critical for the short-term process is made persistently active for up to 24 hours by repeated training, without requiring a continuous signalization of any sort. The kinase becomes autonomous and does not require either serotonin, cAMP, or PKA.

The second and more enduring consequences of the activation of CREB-1 is a cascade of gene activation that leads to the growth of new synaptic connections. It is this growth process that provides the stable, self-maintained syate of longterm memory." 


\section{Motivation $^{2}$}

Motivation is a psychological process which puts puzzling theoretical problems, namely because it's not related to a stimulus that precedes it, but rather to a stimulus or stimuli that follows it. We have to explain how subsequent phenomena can lead us to understand how a proceeding phenomenon is generated.

One of the first attempts to solve this problem was the proposal by psychologists of the existence of the wisdom of the body. A person with a severe adrenal gland lesion requires an exceptionally high amount of sodium chlorite for his wellbeing. It was observed that children in hospital clinics with this disorder ate enormous amounts of salt without requiring any medical counselling for that purpose. This is not a general rule and we don't have specific hungers for many substances of vital importance for the organism, like vitamins.

What happens in general is that a steady state of equilibrium is approached by the body without medical intervention. This is the principle of homeostasis proposed by Claude Bernard (1974) that regulates crucial mechanisms of the body.

McDougal (1913) proposed the existence of propensities to practice behaviors with a specific relevance for the body. We have, then, propensities for eating, drinking, sleeping, resting, having a sexual behavior and perhaps also for social interactions. The problem with these approaches is that the solution proposed makes the problem itself vanish without the requirement for construct explanations.

\footnotetext{
${ }^{2}$ With the collaboration of I. Barahona da Fonseca, Faculty of Psychology of the University of Lisbon and J. C. Tiago de Oliveira, University of Évora, Department of Philosophy, School of Science and Technology, University of Évora, R. Romão Ramalho, 59, 7001-554, Évora
} 
A radical approach has been proposed by ethologists like Nikolaas Tinbergen (1951) and Konrad Lorenz (2002) who invoke instincts as a fix pattern of action, which directs the organism in rigid form to a wanted or convenient end. Nevertheless, not even Sigmund Freud (1920) ever put much weight in the concept of instinct. He did speak of pulsions in a vain, with some similarity to McDougal (1913).

Psychophysiology took another step away, defined physiological needs and invoked psychophysiological mechanisms to correct this needs, or to satisfy this needs.

A competent treatment of psychophysiological mechanisms studied in motivation can be found for hunger and thirst, for example, in Kandel, et al. (2013) and Shagass (1972).

Here remain some epistemological difficulties as for in instance sexual behavior, that does not rigidly obey physiological rules. Psychological and social context have a decisive importance as it may be seen in marriage, fidelity and love in general.

Love tires, creates a state of tension and many prefer to marry substituting the bright colors of love by a grey colorless lack of excitation. A high degree of fidelity, as Søren Kierkegaard (1989) comments, is like going to swim in a small lake loosing, too, the opportunity to swim in the white ocean. The point we are trying to make is that, at a sufficient level of complexity, like dining in an embassy, eating is a social act and the person involved in it has to consult a map in the entrance hall that specifies for him the place at where he must sit during the meal. The sequence of acts of dining in that circumstance is not free and obeys, sometimes, very strict rules of behavior. 


\section{The problem of the reversed sequence of stimuli and responses in motivated behavior}

The problem is far from being simple and the German philosopher Fichte (2005) proposed that one is free to practice all the acts that are necessary for his personality to attain full development of its potentialities - regardless of the heights of the others. This is an anti-democratic kernel that may render impossible a satisfactory respect for the rights of the others.

The essential problem, in itself, is not difficult to be handled theoretically. A need, psychological or in the body, is supposed to generate a state of motivation and this state of motivation guides the organism to attain a final end, which is, in this context, not a cause, but a consequence of the adoption of this central state of motivation. The final structure of the relationship is easily attained without the need of adopting the concept of final causes by means of corrective deviation of the wanted pass way by feedback. If I want a room at a certain temperature during a cold day, I put a heat source in it with a thermometer and an interruption and connection opposite systems in which when the temperature drops, the heat system is put on and when the temperature goes up, the connection system puts the system off.

This example is too simplistic, if we consider human motivations like the drive to attain success or the need to socially accepted, persons enter a complex network of interpersonal relationships. They may wish to make some wanted advancement in the pleasure and satisfaction of his will, but social rules may prevent him to do it, and this is a situation of conflict attractionaversion, or between two attractions or between two versions. The complexity of this system continues in the Freudian architecture of drives and inhibitions, as well as an hierarchy with a supreme moral entity, the super-ego, a working system for practical proposes and a system of basic, strong, affective drives 
which put all of the system under stress and need to be controlled before they lead to a dangerous state of disequilibrium.

Interesting as this analysis may be it is too simplistic. Professionals of psychology, psychiatry, social sciences and alike adopt the method of writing the personal history of the persons they study. This is the entrance of an ideographic point of view substituting bona fide systems approach.

\section{Official and non-official science}

Cultural evolution much before the recognition of psychology, sociology, political economy has advanced examples of personal history, typified in narratives much more sophisticated than our present day psychological counterparts. Readers were prepared to understand the world through these exemplar narratives.

Remember, "Brothers Karamazov", "Crime and Punishment" and Fyodor Dostoyevsky; "War and Peace" and "Anna Karenina" by Leo Tolstoy; "King Oedipus" of Sophocles, "Odysseus" and "Iliad" by Homer, "The Retreat of the ten thousand" by Xenophon, "Antígona" (condemnation due to their abeyance to leaving a tomb to his dead brother); "Exemplar novels" and "Don Quijote de la Mancha" of Miguel de Cervantes Saavedra, "Novels and Pragmatics" by Francisco Quevedo Villegas; Lope de Vega and Pedro Calderón de la Barca, all the works of this authors portrait in a precise form situations of conflict, paradigmatic of essential problems of human condition; François Rabelais and his "Gargantua"; all the French encyclopedists and philosophers of the IIIX century; Montesquieu and his essays; The songs and sonets of Luís de Camões, Shakespeare and his master pieces from love, as "Romeo and Juliet", to horror, like "Macbeth". The futility of Troilus and Cressida, the jealousy of Othello, "The Mercator of Venice", and still 53 Magnificent Sonets to his lover. Poems like "Venus and Adonis", the 
existential conflict of Hamlet, his mother and her lover; the beginning of the romantic era with the suffering of "Young Werther" by Wolfgang Goethe, the lovers swing avant la letter in "Gewälte Verwandschaften"; the historical plays of Historismus and Götz von Berlichingen; his Theory of Color; Faust; we have the great French writers like Honoré de Balzac and "La Comédie Humaine"; "Le Rouge et le Noir", "Chroniques Italiennes" by Stendhal, "Madame Bovary” by Flaubert, "The Maias” by Eça de Queirós, "The Loss of Love" by Camilo Castelo Branco; Émile Zola and "L'histoire Naturelle d'une Famille".. "Ulysses", by James Joyce, The plays from Oscar Wilde, and "The Ballad of Reading Gaol"; "Jean-Christophe” by Romain Rolland, "Les Rougon-Macquart", "Buddenbrooks" of Thomas Mann, "Joseph and his Brothers", "The Magic Mountain", the marvelous novel "Tonio Kröger"; Bernard Shaw plays pleasant and plays unpleasant and an un-social socialist; Henrik Ibsen, August Strinberg; the marvelous present day French writers, like "l'Espoir" and "La Condition Humaine", from André Malraux. "Justine ou les Malheurs de la vertu", Marquis de Sade and "Les liaisons dangereuses" by Laclos. "Tales of Mystery and Imagination”, Edgar Allen Poe, "Tropic of Capricorn" by Henry Miller and "Quiet Days in Clichy". "Le vicaier and soldiers" and "Schriftversteller" by Hochhut, "Lady Chatterley's Lover" by DH Lawrence; “A Streetcar named Desire”, by Tennessee Williams and "The Grapes of Wrath" by John Steinbeck. "Twenty Thousand Syrians" by Truman Capote, The great poets Fernando Pessoa, José D’Almada Negreiros, Paralelo 42, John dos Passos, Mário de Sá Carneiro, José Régio, Miguel Torga, Maria Gabriela Llansol and Herberto Helder and Alexandre O’Neill.

We are not specialists in literature and this quotations serve only to defend our point of view that an enormous bulk of knowledge exists beyond science, and may serve to prepare man for a competent co-cultural judgment and a complement to strictly scientific cultural formation. 
The ideographic approach can be substituted by the nomothetic one, but changes in the co-efficient of the corresponding system equations will make them non-linear and render them difficult to deal with from a mathematical analytical point of view.

Returning to the nomothetic point of view, we must first consider that the origins of motivated behavior are the internal stimuli be they vegetative, emotional or cognitive.

We have then:

$$
\left[\begin{array}{c}
x_{1} \\
x_{2} \\
\vdots \\
x_{n-3} \\
\text { int }_{\text {var }} \\
\text { inc }_{\text {var }} \\
\text { rec }_{\text {var }}
\end{array}\right] \text { Ref }{ }^{n \times n} \quad M \equiv Y(t+1)
$$

We have an operator, motivation, "Motive", which is more or less strong, which is expressed dimensionally by the value of "Drive"; The incentive, "Incursive Stimuli" and "Recursive Stimuli" are represented by "Inc s" and "Rec s", respectively. Locomotion will represent both the walk of the organism in the space of phases or else in a conceptual space of cognition.

When Drive attains a certain intensity, taking into account the incentives, a motivated behavior will start. It will be prosecuted in successive cycles separated by the end of $\operatorname{Div}=\operatorname{Ref}-Y(t) \quad$ (divergence from reference) which marks the correction of distortion during the active part of a motivation cycle. Note that similarly with what happened to the concept of habit in learning, we have now in motivation the concept of intention.

In other words, intention is defined in this context as the construct that links together the entities we have just mentioned. When this structure acts on 
the concrete values of the above mentioned variables it produces the next intended state, as well as in the long run of activity, the pass way that leads from start to the aimed end. 



\section{Affective Processes ${ }^{3}$}

We start from the perspective in which affective processes are considered the most basic propositional formulation for attribution of value. From a pragmatic viewpoint they play a decisive role in determining and regulating the relationships, not only between persons but also between states. The rules regulating them should be those that might assure legitimacy and optimality. It can be thought that it would be a decisive advancement for International Policy if the United Nations adopted a code for relationships between nations and obtained unanimous consent in their acceptance.

We can ask ourselves if ethical or value codes must be founded, in value or ethical propositions, or if, on the contrary, value and ethics could be determined after propositions of fact. Strawson (1952) asserts that conclusions of value can never be deduced from premises of fact, and, therefore, here exists a guideline for the choice of founder principles of a theory of value. It could be argued that true premises have the same value as false premises, in a value theory. Nevertheless, in a true premise, the ostensive value of truth is present, while in its negative counterpart the situation is more complex. Mainly, quoting Charles Sanders Peirce (1931-35), if we say that something, for instance an attribute, is not true about a certain entity, it means that another alternative attribute has to be true. For example, in the proposition in which we say "The color of the sky is not blue", it is implicitly asserted that it has to be of another, alternative, color, for instance, in a winter day. The truth value and the referential value are distinct in the two cases, the assertive and the negative.

\footnotetext{
${ }^{3}$ With the collaboration of I. Barahona da Fonseca, Faculty of Psychology, University of Lisbon
} 
Historically, in the XIX century, it was asked if encephalon structures implied in the occurrence of emotions, feelings, moods and alike should be the same as those involved in the processing of purely conceptual nonaffective intellectual thinking.

In a brief summary of the main theories proposed for the explanation for affective phenomena, we can mention alternatively and in some complementary form Head's (1920) theory, that proposes that the affective quality is due to the fact that sensory information impinges on thalamus and hypothalamus and in general the limbic system, that informs the brain, and subsequently, the vegetative, endocrine and somatic systems; Yet, a complementary point of view is carried by the proposal of James-Lange (1884), who think that stimuli are carried to the vegetative and somatic periphery and thereafter they are re-informed to the brain. The affective quality would result from the affective quality imposed by the vegetative system on sensory data - I'm sad because I cry - alternative to - I cry because I'm sad. In modern versions, explanations became more complex. In his theory, Lindseley asserts, as a decisive component, the excitation or arousal of the brain, which is then interpreted cognitively according to the experience a subject may be having, independently, but in an associated form. For instance, the injection of adrenaline would provoke excitation. The interpretation of this excitation would be as expressing sadness if the context in which it is felt is sad and pleasure if the context in which it is felt is pleasant. Finally, in Magda Arnold's (1960) theory, the analysis of facts is even more complex, and implies the interplay of different structures, like the amygdala, the hippocampus, the limbic cortex and finally the frontal cortex and telencephalon in general. It should be noted that an emotion is an acute state with a positive or negative valence, which is associated to a state of arousal. This brief phenomenon is then further elaborated by the more 
complex cortical structures, giving rise to feelings which imply the occurrence of complex cognitive components.

\section{Some reflections on the nature of affective processes}

Starting our analysis from this data, we have to recognize that the basis for affective processes is heterogeneous. It implies censured, vegetative and somatic, memory, cognitive processes, as well as expression of inner states and incentives for action, directed to others involved in the affective process.

As in the case of learning, although in another sense, convergence phenomena are present and represent a stress imposed in an intellectual structure, requiring efforts to the resolution of conflicts of interest or concurrences of means to attain ends.

\subsection{The quantum structure proposed for emotion}

Let us consider the set of proposed stimuli $s_{1}, s_{2}, \ldots s_{\mathrm{n}}$, respectively. Together with motivations $m_{1}, m_{2}, \ldots m_{\mathrm{n}}$, a process of constitution of relationships that is multi-modal, and which integrates different levels of functioning in the organism, which can cause disarray in subjects going through an intense state of emotion, and finally, being accosted for psychiatric disturbance.

We can then define different structures at different levels, namely:

$$
\begin{gathered}
H\left(s_{1}, s_{2}\right) H\left(s_{1}, s_{2}, s_{3}\right) \ldots H\left(s_{1} \ldots, s_{n}\right) \\
H\left(r_{1}, r_{2}\right) H\left(r_{1}, r_{2}, r_{3}\right) \ldots H\left(r_{1 \ldots}, r_{n}\right) \\
m_{1}, m_{2}, m_{3} \ldots, m_{n}
\end{gathered}
$$


Means to attain ends can be defined as Sequence 1, Sequence 2..., Sequence $n$, incentives $i_{1}, i_{2}, \ldots, i_{n}$ enter as a title analog to drive.

These are components of a transitory organization, which corresponds to the change of state which is associated with an emotion of an affective state in general. Maybe felt as pleasant or unpleasant, it is always given a value as if it belonged to another organism.

In chemistry, valence processes give a structure with resilience to chemical entities. In psychology, the psychological organization of reactions, sensory processes and motivation strategies impose combinations as well as exclusions.

For instance, suppose the problem raised by someone we love and is angry at us. It provokes her rage $(r a)$, and simultaneously a not angry state $(n a)$ in our part, which corresponds to:

$$
H_{r a}(s, r)
$$

And from our part:

$$
H_{n a}(s, r)
$$

And stimuli evaluation as disagreeable $(s \rightarrow d)$

$$
d_{1}, \ldots d_{n}
$$

On the enraged loved one and on the angry an attenuated reaction $(r \rightarrow$ $\left.d^{\prime}\right)$ :

$$
d_{1}^{\prime}, \ldots d_{n}^{\prime}
$$


Which are integrated in a complex structure of motivation $m_{1}$ (for subject one), and $m_{2}$ (for us).

The contenders for the fight are in place. If there is an intention of a pacific solution, for both contenders, $m_{1} \cdot m_{2}$, takes place and the solution is $m_{s}$ for both contenders.

Now, $d$ and $d^{\prime}$ disappear and are substituted by $d_{s}$ and $d_{s}^{\prime}$ for all the components of the conflict.

Now we can write the final solution:

$$
\frac{d H_{1}\left(d_{s}, d^{\prime}\right)}{d t}=H_{2}\left(d_{s}, d_{s}^{\prime}\right)
$$

We can, in this approach, represent subjects as complex structures with established complex relationships, with their counterparts in other subjects. That means that some principles of anti-symmetry have to be respected if the integrity of contenders in human conflicts is desired and promoted. What happens here, the Hermite transform of the second type, is the information available concerning each one of the individuals involved in the interaction. This Hermite function has multiple components and a principle analogous in some measure to Pauli (1925) exclusion principle has to be found if the integrity of individuals and participants in social conflicts is needed and must be kept within limits, otherwise uncontrollable conflict and disease may occur.

A remarkable quality of emotional components is that they have a structure that implies that a person is conscious of what is happening and is actively evaluating it.

This is extensively present in the Lindseley-Shachter [ (Emotion, 1951) (Schachter \& Singer, 1962)] paradigm and in an implicit form in the JamesLange [ (James, 1884) (Lange, 1922)] paradigm. 



\section{On Cognition ${ }^{4}$}

Cognitive processes were rigorously described by Wolfgang Köhler (1976), using data obtained in a research performed in Canary Islands.

The experiment was a very elemental one and consisted in maintaining primate monkeys in a cage with bars, spaced enough so that animals could touch objects placed in the exterior of cage. In the floor of the cage animals could find a stick in wood. In the exterior bananas were offered, but at a distance that the animals couldn't reach. Without hesitation animals picked the stick and with it approached the bananas to the cage so that they could eat them. Apparently without learning, and any failed attempt, animals were immediately capable of having a satisfactory meal.

The important difference to conventional learning experiments was that there was no approach phase during which the animals were progressively solving problems caused by the successively difficulties. It was as if animals solved their problems in an interior space, and then behaved according with the solution found by their intuition.

Naturally this was immediately taken as a paradigm for many human situations, in which learning is also immediate, not needing any rehearsal of the necessary procedures.

\section{The mathematical paradigm}

We choose, as a representation for this mathematical paradigm, the delta wave of Dirac (1981). Dirac's delta wave has zero value if:

\footnotetext{
${ }^{4}$ With the Collaboration of Roberto Moreno-Dias, University of Las Palmas de Gran Canaria and J. C. Tiago de Oliveira, University of Évora, Portugal
} 


$$
\begin{gathered}
\delta(x)=0 \text { if } x \neq 0 \\
\delta(0)=\infty \\
\int_{-\infty}^{\infty} \delta(x) d x=1
\end{gathered}
$$

The second reflection concerns the fact that any function $f(c)$ of frequency $v$ is representable by a Dirac's comb, with repetition with a frequency $v$ of Dirac's wave.

Given any function of the Central Nervous System (CNS), $f(t)$, cross corelation between $f(t)$ and Dirac's comb will produce the components $f v(t)$ of $f(t)$. This way we attain the most basic level of representation of events in the CNS. Campbell (1969) used bi-dimensional sinusoids to represent any picture of the reality with two dimensions. This is not surprising as trivial fourier analysis allows the decomposition of a sound in time, in an infinite set of components. As it is available the domain of space and time, here we have for both the domain of frequency.

In a system with convolution function $\int h(t-\tau)$ the product of this expression by $f(t)$ has an output which is represented by:

$$
Y(\omega)=f(\omega) \cdot h(\omega)
$$

In which $h(\omega)$ is the transfer function of the system and $h(t-\tau)$ is the convolution function. Messages between different points of the CNS convey information, for instance, frogs make sexual appeals, which have specific codes of frequency. On the other hand, the analysis of stimuli is closely related to behavior. Lettvin, Maturana, Pitts and McCulloch (1968) identified neurons that reacted specifically to food or to predators. There, identification was expressed in specific modes of reaction - namely, when in the screen in 
front of them was projected a black circle which moves slowly, and which suffers dilatation when the circle attained a certain diameter, the frogs jumped, trying to catch it as if it was an animal.

On the other hand, when a sudden darkening over the frog happened, it jumped - trying to escape from that area, as it would happen if a predator has opened his wings in a stalk reaction to catch the frog.

These examples are of little help to build a theory of cognition. Remember that human beings deal with abstract symbols that stand for other objects, representing them, and are not themselves the symbol of the thought. Charles Sanders Peirce (1931-35) categorizes human symbols in Icons, Diagrams and Signs. Icons like in images of the orthodox Christian represent particular characteristics of the face or the body of the saints trying to express some specific character for devotes; Diagrams have correspondence in architectural plans where there exists no exact correspondence between the proportions of the scheme which is drawn and the building which is to be implemented and finally the sign which can be interpreted as a word, in which the components and the meaning are completely arbitrary, and require a theory to deal with symbols, components, syntax and semantics. The meaning of the phrases in a common language is more difficult because it's not rigidly specified and depends on the understanding that the reader has of the symbols, words, and morphemes and of the syntax and the semantics. No theory of cognitive knowledge is possible for man without an adequate theory for language. The efforts to create an adequate theory for language began in 1910 with Ferdinand de Saussure (1959), who distinguished between Langue, Langage and Parole, that is, the commonly used way of communication, the rules of it, and the way subjects produce expressions to be communicated. Poetry has a departure point. Let us consider a sophisticated poem in 
Portuguese by Fernando Pessoa's heteronym Ricardo Reis (1946), which reads as follows:

Para ser grande, sê inteiro: nada

Teu exagera ou exclui.

Sê todo em cada coisa. Põe quanto és

No mínimo que fazes.

Assim em cada lago a Lua toda

Brilha, porque alta vive.

To be great be all yourself

Nothing yours exaggerate or exclude

Be all yourself in each thing

Put whatever you are in the minimum you make

So, in each lake the entire Moon shines

Because high it lives ${ }^{5}$

Reading this poem, it appears that it's possible that a repetition occurs, but this is not the case. The first verse puts straightforward the requirements of a complete involvement of the subject in everything. The second verse speaks about the subject, describing it, not as involved in an action, that is "Nothing yours, exaggerate or exclude", but as a statement "Be all yourself in everything you do", which is a behavioral requirement for an entire evolvement of the self (it opposes the ego to the self). "Put whatever you are in the minimum you make". How much? Always. Since it is the minimal condition for it. So, in each lake, independently of its dimension, the entire moon shines because high it lives. We will give another example of this type of analysis:

\footnotetext{
${ }^{5}$ Translated by the author
} 32 
Wish little, you will have everything;

Which nothing, you will be free.

The same wish they have for us, wishes us

Oppresses us

The representation of the poem's content. How does the poet perform his task of translating his cognition into words? Let us consider the logical relationship between the words.

In what follows, the symbol $\otimes$ represents a logical operator whose terms are two mathematical objects; each one of these objects is associated with a semantic content.

\section{Wish little}

Reduce wish or renounce to wish - you will have everything $\otimes$ wish nothing, you will be free.

\section{The same wish they have for us}

The same wish with which others favor us, requires counterparts $\otimes$ oppresses us

Final discussion, we attain in this way a plausible, competent manner of representing meaning in very sophisticated and complex structures. Notice that we have here to answer some questions. If I have the qualification by a quantifier and trough an operation or multiplication I make it fade, as for instance "Wish little you will have everything", the truth is that I have produced a new meaning. It is not that the first is false, if I say Wish nothing you will be free, again wishing nothing does not result necessarily that you will be free, it is only an approximation to a meaning. We are in a system of 
representation, fuzzy logics used by Zadeh (1965) to characterize the amount in which a certain entity processes a quality.

Our difficulties don't end here. Having different persons a different understanding of language, how can I be sure that they are correct, when with different words they assert they are telling the same thing? The answer is required for all the system we've been building. The plain truth is that we live inside a glass of equivalence of functions or, what is the same, we use invariants to communicate. And now begins another problem. How can we be sure that the invariants I use to communicate are the same as those you use to understand me? 


\section{Social Processes ${ }^{6}$}

All the interactions, as well as the components of systems of lower level of complexity in the sense of Herbert Spencer, are present in collective organizations and require an analysis that encompasses the social level. There is, nevertheless, a need for the recognition of an instrumentality of the less differentiated processes, relatively to the higher ones, either cognitively or from a pragmatic and instrumental point of view. It is inherent to the social interaction that the system's components are allowed to use natural and colloquial language in all their interactions, as well as the most differentiated cognitive processes, the most refined and extensive forms of long term memory, the most demanding rules of ethics, required for politics and for the uses of science and art.

It is not the case that the most basic psychological processes are not present, and even the bodily processes and their experience in survival, whose experience is needed to survival, adaptation to the environment, even for the most elementary forms of experience of the self. From a phenomenological point of view, the self is experienced in an implicit latent manner, reflexive components are also present but mainly under a disturbed condition. Basic experiences of the self are self-directedness and self-ownership in the relation with the environment we have to consider meaning and intention. In Martin Heidegger's (1980) approach, the meaning of a scissor is given by our fingers movements and the movements of the scissor when it cuts. Heidegger speaks in this case of meaning depending from a handlich kite. In what concerns intention, the problem is more complex: let us suppose that an organism function is regulated by the following equation:

\footnotetext{
${ }^{6}$ With the collaboration of J. C. Tiago de Oliveira, University of Évora, Portugal and Paulo Castro, Centre for Philosophy of Science of the University of Lisbon
} 


$$
I(t) \oplus N S(t) \equiv Y(t+1)
$$

So that the future output state $Y$ depends on the input $I$, the present state $S$, and the state transition matrix $N$. $I(t)$ contains recursive variables $t-k$ to $t-1$ to arbitrarily $v(t+l)$ which are incursive variables, which concern the future, as it was proposed by Daniel Dubois (1998): “This consideration of the future gives to the relational structure a character of a fractal."

The nature of this conceptual structure is one in which conceptual and cognitive processes are the individual domain of the considered functions and social interactions related to them are their counter domain. They don't exist one without the other, but not withstanding this condition, the counter domain processes depend exclusively from the original domain processes. There is an abstract relationship which transforms them in both senses.

We must not loose, even at this superficial level of analysis, facts which are crucial to the characterize phenomena considered both at an individual as well as a collective perspective. In the social perspective, the new quality that data has acquire expresses itself in the operations of appropriation or alienation of the acts or psychological processes of the self or others in the formation of the more varied collectives.

In an extreme condition, I can substitute a part of mine that does not function by a part of another that does, this way creating a prosthetic dynamic process of social nature that comes to existence. I can also eliminate or alienate another personal process that I do not wish to possess or exhibit.

In certain cases I can alienate it in another person, as it happens in the creation of value in work processes in which one appropriates the value of his work in another that then appropriates it without equilibrium to the rights of the first. This two opposed principles of appropriation and alienation do occur 
in two general contexts - the context of cooperation and the context of controversy.

The interactional contexts of cooperation and conflict are regulated by political structures, such as the Constitution, the rules of access and use of power, the distribution of power by the individual and constitutionally specified structures of a society.

Complementary to this, the individual agents with social intervention will aggregate according with economic rules, with pragmatic and social utility, which includes scientific, artistic value, access to a competence, or simple self-organization as well as the culturally sanctioned rules of welfare, from the historical permissible to the historically unacceptable. As a main source of power, in our western societies, we have the creation of wealth as well as its distribution by the different social classes.

Their knowledge and possession are subjects of dispute and attempt of possession, and these two aspects are motives for controversy and dispute.

In non-democratic authoritarian regimes, many times the conflicts have their source and motivation the dispute over the control of these mechanisms. On the other hand, there is a constant conflict between permissible strategies of appropriating power and richness and the creation of protection against these moves using regulations. Many times and in many circumstances the appropriation of power, if necessary by the use of coercive measures or economical strategies in order to favor a given class against the interest of others, are the single motive for disguised or ostensive strategies of intervention.

It is not here the place to analyze this social phenomenon. We will only formalize the phenomena of response production with their laws of adaptation, similar to habit formation, intervention of drive and motivation, cycles an oscillation and uses of information for decision making. 
Remember that phenomena at this level of description have a dual status (1) as an expression of the individual non-social domain and (2) as members of the social counter-domain. The first, to the learning, motivational and intellectual ways of decision making, the second, as a mean to attain ends at a social level. 


\section{The Hodgkin Huxley study of action potential $^{7}$}

Hodgkin and Huxley (1952) studies of the squid axon using the technique of clamp voltage in stimulation and recording. They cut a small portion of a squid axon, inserted a conductor wire inside of it, between the two tops and sealed the two tops of the segment of axon, they made this to guarantee that all of the axon is at the same potential. Then, they introduced a stimulator electrode linked to a current generator and a recording electrode that measured the potential differences between the outside and inside membrane of the action of the squid. They verified that at rest, the interior of the axon is at the potential, about $70 \mathrm{mv}$ lower than outside the axon. At rest, this membrane potential depends almost exclusively from potassium, under Nernst equation, is given by:

$$
V m=\frac{R T}{F} \log n \frac{\left[K^{+}\right] \text {outside }}{\left[K^{+}\right] \text {inside }} \cong-70 m v
$$

During state of rest the permeability for $\mathrm{Na}^{+}$is approximately null, so, during rest, the contribution of ions $\mathrm{Na}^{+}$for the membrane potential is scarce. In normal states of cells, when the state of rest is altered and the neuron is activated this occurs through the effect of Excitatory Post-Synaptic Potential (EPSP), which depolarizes the membrane making it more positive relatively to the outside of the membrane.

${ }^{7}$ J. Barahona da Fonseca, Faculty of Sciences and Technology, New University of Lisbon; I. Barahona da Fonseca, Faculty of Psychology of the University of Lisbon and J. Simões da Fonseca, Faculty of Medicine of the University of Lisbon 
When a threshold is attained, the channel for $\mathrm{Na}^{+}$opens and allows the penetration of massive quantities of $\mathrm{Na}^{+}$. As a matter of fact, this increase in the permeability for the $\mathrm{Na}^{+}$, augments multiplicatively about 900 times, which provokes a depolarization which grows rapidly and exceeds the 0 difference of potential of the membrane, whose potential is now about $+40 \mathrm{mv}$ inside to outside of the cell membrane, which is the equilibrium potential for the membrane during activity and which is the result of the opening of channels rendering the membrane potential dependent of $\mathrm{Na}^{+}$. This equilibrium potential for $\mathrm{Na}^{+}$is now given under the Nernst equation.

$$
V m=\frac{R T}{F} \log n \frac{\left[\mathrm{Na}^{+}\right] \text {outside }}{\left[\mathrm{Na}^{+}\right] \text {inside }} \cong+40 \mathrm{mv}
$$

Hodgkin and Huxley studied the variations in the fluids outside the axon by measuring radioactivity of marked ions and calculated the flux of ions according with the changes in potential produced in the cell, in the interior of the axon, by means of a current generator. They were this way able to write the equations for:

$$
\begin{gathered}
\mathrm{Na}^{+} \mathrm{K}^{+} \\
\mathrm{Cl}^{-} \& \mathrm{Ca}^{++}
\end{gathered}
$$

So they described the generation of an action potential according to differential equations. The model was tested and co-efficiently adjusted to produce a reproduction of the phenomena observed experimentally.

The differential equations which describe the process will be next presented. We only advance that during the generation of an action potential $\mathrm{Na}^{+}$penetrates massively and then more slowly, on the contrary $\mathrm{K}^{+}$flows to 
the outside with an increasingly strong current, until both reach a peak. Then, the permeability to $\mathrm{Na}^{+}$ceases and for $\mathrm{K}^{+}$continues for a while.

The combination of the effect of these two factors makes the action potential drop to the rest level. It follows an absolute refractory period and afterwards a relative refractory period for the generation of an action potential. Note that we explained all the ionic changes by means of ion concentration and electro chemical factors alone. Now we have the problem posed by an excess of $\mathrm{Na}^{+}$inside the cell and an excess of $\mathrm{K}^{+}$outside the cell. A Sodium $\left(\mathrm{Na}^{+} / \mathrm{K}^{+}\right)$pump begins to operate, to extrude $\mathrm{Na}^{+}$and intrude $\mathrm{K}^{+}$. This process can be seen as a substance $x$ links itself in the exterior of the cell to $K^{+}$. Then it is more concentrated outside than in the inside, and due to this gradient, penetrates the cell. Inside the cell, it liberates $K^{+}$and this transforms into $y^{+}$substance that links itself to $\mathrm{Na}^{+}$, and as it is more concentrated inside than outside it goes through the membrane and liberates $\mathrm{Na}^{+}$outside. This is already an active process that cannot be completely explained in electro chemical terms, and requires consumption of energy.

In a rigorous form, what has happened with this approach was that they utilized squid preparations with axons that measured $1 \mathrm{~mm}$, which were visible from the naked eye. They used two technics which were relevant to the study, namely the spatial clamp and the voltage clamp. The spatial clamp consisted in a metallic wire which forced a potential uniformity alone the axon segment which was being studied. On the other hand, two pairs of electrodes, one in the exterior and the other inside of the squid axon, allowed the introduction of a current by one pair, and the measurement of the results by the other pair of electrodes.

The Goldberg equation, which relates $\mathrm{Na}^{+}, \mathrm{K}^{+}$and $\mathrm{Cl}^{-}$concentrations with membrane potential is expressed by: 


$$
V=\frac{R T}{F} \log \frac{p_{K^{+}}\left\lfloor K^{+}\right\rfloor_{\text {out }}+p_{N a^{+}}\left\lfloor N a^{+}\right\rfloor_{\text {out }}+p_{C l^{-}}\left\lfloor C l^{-}\right\rfloor_{\text {in }}}{p_{K^{+}}\left\lfloor K^{+}\right\rfloor_{\text {in }}+p_{N a^{+}}\left\lfloor N a^{+}\right\rfloor_{\text {in }}+p_{C l^{-}}\left\lfloor C l^{-}\right\rfloor_{\text {out }}}
$$

Hodgkin and Huxley, in their experiments, varied the concentration of sodium ions outside the membrane. Excluding extra-cellular $\mathrm{Na}^{+}$, it was possible to measure the contribution of $K^{+}$to the membrane potential. Measuring the current observed in the exterior of the cell membrane, they determined the contribution of the sodium ion. They also confirmed that these two ions had the most significant contributions for the potential of the membrane. The hypothesis of Hodgkin and Huxley is that an axon segment may be represented by an equivalent circuit.

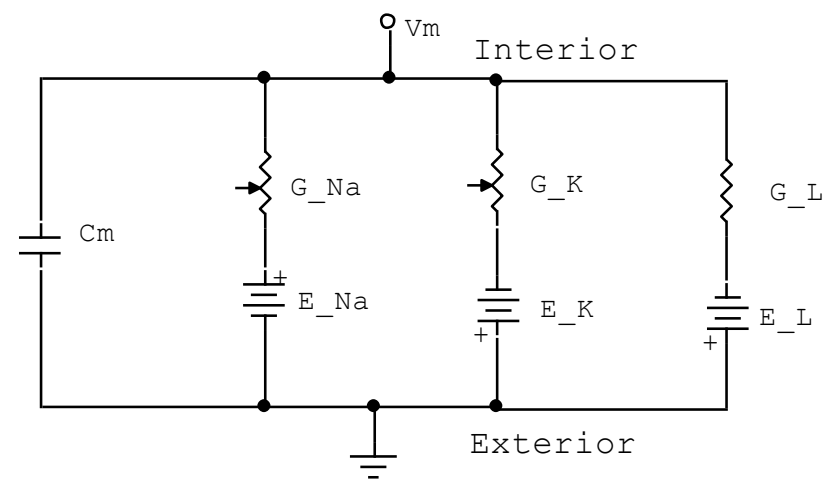

Fig. 2 Model of the Circuit Equivalent to the Membrane Capacitance, resistivity and inductance

In this model the current which crosses the membrane has two main components: the capacities and ionic current. The last component has itself three sub-components: the $\mathrm{IK}^{+}, \mathrm{INa}^{+}$and $\mathrm{ICl}^{-}$. The differential equation describes the phenomena which occur in the equivalent circuit. 


$$
C_{m} \frac{d V_{m}}{d t}+I_{\text {ion }}=I_{\text {ext }}
$$

Where $C_{m}$ is the membrane capacitance, $V_{m}$ membrane potential, $I_{\text {ion }}$ for the total current due to ionic contribution, and finally $I_{\text {ext }}$ stands for a current externally applied. $I_{i o n}$ is given by:

$$
I_{\text {ion }}=G_{N a}\left(V_{m}-E_{N a}\right)+G_{K}\left(V_{m}-E_{k}\right)+G_{L}\left(V_{m}-E_{L}\right)
$$

Each Ionic component $I_{i o n}$ has a characteristic conductance associated $G i$ and equilibrium potential $E_{i}$.

In the Hogkin-Huxkey model, the ionic contributions assume the following form:

$$
I_{\text {ion }}=\sum_{k} I_{k}=\sum_{k} G_{k}\left(V_{m}-E_{k}\right)
$$

The fundamental hypothesis of Hodgkin and Huxley was that the ionic conductance $G_{N a}$ and $G_{K}$ did variate dynamically with the membrane potential $V_{m}$. This dynamic variation was dependent of the physical characteristics of the channels, designated by voltage gated ion channels.

Nowadays, the channel conductance for each ion is differentiated from the global conductance of the membrane. The macroscopic conductance for the different ions can be considered as the sum of a great number of microscopic channels. Each microscopic channel can be conceived as possessing a number of gates for the passage of ions. Each individual gate can be in one of two states: permissive and not permissive. Only when all the gates in one microscopic channel are open can ions traverse that channel. Hodgkin and 
Huxley introduce a probabilistic relation between the opening of channels and the membrane potential. This way, the probability that a channel is in a permissive state depends on the membrane potential.

$p_{i}$ is a channel probability to be in a permissive state. Considering a number of channel we can define $p_{i}$ as the relationship between the number of permissive channels and the total number of channels of the same kind. The transition between the permissive state channels and non-permissive state channels follows the relationship:

$$
\frac{d p_{i}}{d t}=\alpha_{i}(V)\left(1-p_{i}\right)-\beta_{i}(V) p_{i}
$$

In which the variables $\alpha_{i}$ and $\beta_{i}$ define the rate of transition between permissive and non-permissive states. The membrane potential will stay stationary while the state of the gates is also stationary, (i.e., $d p_{i} / d t=0$ ) as $t \rightarrow \infty$ is given by:

$$
P_{i, t \rightarrow \infty}(V)=\frac{\alpha_{i}(V)}{\alpha_{i}(V)+\beta_{i}(V)}
$$

The time which is necessary to reach $63 \%$ of this equilibrium value is given by:

$$
\tau_{i}(V)=\frac{1}{\alpha_{i}(V)+\beta_{i}(V)}
$$

The macroscopic conductance for a set of channels is proportional to the number of channels in a permissive state which in turn is proportional to the number of channels in which the gates are in a permissive state. 
This way, the microscopic conductance of Ion $K^{+}$due to channels type $K^{+}$ with constituent gates type $i$ is proportional to the product of the probabilities that the individual gates are open, $p_{i}$

$$
G_{k}=\bar{g}_{k} \prod_{i} p_{i}
$$

Where $G_{k}$ is normalization constant which determines the maximum possible conductance when all channels are open.

Hodgkin and Huxley modelled the sodium $\left(\mathrm{Na}^{+}\right)$using three gates of the so called $m$ type and one gate of the $h$ type. Applying the last equation to the sodium channel $\left(\mathrm{Na}^{+}\right)$, we obtain:

$$
G_{N a}=\bar{g}_{N a} p_{m^{3}} p_{h} \equiv \bar{g}_{N a} m^{3} h
$$

In the same way, the $k^{+}$conductance is modelled with four similar gates called $n$.

$$
G_{k=} \bar{g}_{K} p_{n^{4}} \equiv \bar{g}_{K} n^{4}
$$

In the Hodgkin and Huxley model the electric currents are given by:

$$
\begin{gathered}
I_{\text {ion }}=\bar{g}_{N a} m^{3} h\left(V_{m}-E_{N a}\right)+\bar{g}_{k} n^{4}\left(V_{m}-E_{k}\right)+\bar{g}_{L}\left(V_{m}-E_{L}\right) \\
\frac{d h}{d t}=a_{h}(V)(1-h)-\beta_{h}(V) h \\
\frac{d m}{d t}=\alpha_{m}(V)(1-m)-B_{h}(V) m
\end{gathered}
$$




$$
\frac{d n}{d t}=a_{n}(V)(1-n)-\beta_{n}(V) n
$$

In order to determine how the six state transition velocities depend on the membrane potential, we consider the equations (14) together with the equation (5), completely specifying the membrane potential $V_{m}$.

Hodgkin and Huxley obtained the rest state conductance for $V_{m}=0$ and then applied currents produced by the current generator that produced a potential $V_{c}$ and determined the time necessary for the conductance to attain half of its maximum value for a given $V_{c}$.

The difficulty that data posed to attain concordance that would satisfy in a sigmoid form of the growing of the conductance led to the verification that the initial phase of slow growing, followed to a much faster growing is obtained in an exponent of $p=4$ as it can be seen in the following equation:

$$
G_{k}=\left\{\sqrt[4]{G_{\infty}\left(V_{c}\right)}-\left[\sqrt[4]{G_{\infty}\left(V_{c}\right)}-\sqrt[4]{G_{\infty}(0)}\right] e^{-t / \tau_{n}}\right\}^{4}
$$

John Eccles (1957) has extended Hodgkin and Huxley theory about the axon to the entire neuron. He verified that conditions of the axon do also apply to the entire cell, namely dendrites and cell body. Ricardo Miledi (Miledi \& Slater, 1966) studied specifically the role of the trigger lock in the axons hillock in the generation of patterns of axon sequences that possibly correspond to different content messages. 


\section{An alternative model: The Quantum Mechanics Model of a Particle against a Potential Field}

There are cases in which the very satisfactory model of Hodgkin and Huxley cannot be applied. This happens, for instance, in the case of Horizontal Cells in Retina which are stimulated by electrical synapses. This means that there is no ionic change of conductance during, at least, the initial post-synaptic phase of generation of the action potential.

We can putatively admit that the flow of electrons comes against the negative field which corresponds to the interior of the cell.

This means that electrons are repelled if their potential is inferior to the negative barrier potential and passes through if it is negative to the rear potential of the cell.

This was the classic understanding of the case a particle against the potential barrier. In Quantum Mechanics it becomes different: even when it has a potential lower than the barrier potential, a tunnel phenomenon makes particles appear inside the barrier with a non-negligible probability and traverses the barrier with a velocity much higher than the velocity of light until particles are extruded from the tunnel condition.

This way, we have a nervous system working at a slow velocity of Ion Changes which produced propagations along axons of the order of $140 \mathrm{~m} / \mathrm{s}$ and in cases it occurs in a cable conduction way with a speed of the order of the speed of light.

This way particles which traversed the potential field, when they attained a cable condition, for instance, axons insulated by sheets of myelin are conducted in a cable way, satisfying the conditions of cable conduction: high velocity with exponential potential decay, due to the fact that myelin does not allow ion conductance across the cell membrane. 
Myelin covered membranes are interrupted by $1.5 \mathrm{~mm}$ ranvier nodules, covered by naked axonal membranes.

When the impulses arrive at these nodules with diminished amplitude, to the exponential decay in cable conditions, they are still high enough to trigger an action potential at the entrance of the naked ranvier nodule. Then they go across this nodule in a electronic way and trigger a action potential when they enter again a segment of axon covered by myelin.

José da Fonseca (1978), using this paradigm, proposed that in the case of cells attained by a flow of electrons, the same does occur and a estate of activity is generated. Horizontal cells in retina work with this conversion of electrons flow into ionic changes. This cells which exist in retina have an effect in the modulation of activity in the ganglion cells which connect retina to lateral geniculate nucleolus.

This renders available to, for instance, colliculi superiores, a very fast representation of the state of retina. This is a very great advantage for adaptation to the environment because it allows the use of a representation much more quickly than the environment can effectively change.

This does not contradict Hodgkin and Huxley (1952) results and achievements but rather complements them, and can be used to explain in some conditions how a change of state which is relevant for learning, can be attained, without repetition, in an almost instantaneous way, as it occurs in some cognitive forms of learning. 


\section{Attention $^{8}$}

Just at the beginning of World War II, Dempsey, Morison (1943), and Magoun (1952) discovered that stimulating the midbrain reticular system the attention of animals was enhanced. On the other hand Fuster (1980) made an experiment using clicks to provoke acoustic evoked potentials. During that experiment, an amplitude reduction of acoustic evoked potentials was observed in a cat that was confronted with a rat placed inside a glass bottle.

This was interpreted as meaning the reticular system was controlling the attention of the cat to the hearing clicks. In Richard Jung (1958) laboratory it was demonstrated that stimulating the mid-brain of brainstem of cats the stress hold for critical flicker fusion was elevated and responses to neuro-cells to light stimuli were also enhanced, and this was interpreted as a change in attention.

Vigilance was the process which began to be studied, being at the maximum during attention and minimum during deep sleep and coma.

The use of an electroencephalogram allowed identification of different levels of vigilance. During the awake state with eyes closed, the brain showed an approximately 8 to 9 cycles c/s alpha rhythm. When falling asleep, first, EEG became flat with a reduction in alpha rhythm. In a second phase, sleep spindles appeared, 8-12 cycles c/s in frequency, and great K complexes. When sleep was deepening to a middle level slow sleep a theta rhythm, 4 to 7 cycles per second increased in amplitude. Thereafter, delta waves 1 to 3 cycles per second increased and substituted theta waves. To these successive states of function of the brain corresponded to a successively deeper state of sleep. Michel Jouvet (1958) identified paradoxical sleep - a sleep that in appearance

\footnotetext{
${ }^{8}$ With the collaboration of Paulo Castro, Centre for Philosophy of Science of the University of Lisbon
} 
was not distinguishable from a state of arousal, characterize by beta waves 14 and higher cycles per second, but that appeared together with rapid eye movements - REM sleep, which was also associated to loss of muscle tonus and irregularities in cardiac rhythm.

So, during REM persons dream, and there aren't, generally, any movements, although in older persons, in which the reticular system is damaged, there appear states of physical agitation.

In general, nightmares appear associated with REM sleep and sleep terror associated to slow theta sleep. If someone tries to awake a person during slow sleep can be attacked with violence.

A singular discovery was that tricyclic chemical agents, very efficient in the treatment of depression made REM states disappear. Sleep deprivation can be very disagreeable and needs to be corrected after a short while. Modern political interventions imply the use of sleep prevention during very large periods, of ten days or more. We observed one case of more that 15 days. In this case, persons become very upset and need a long time for recovery. In some cases it may be that they never do recover again.

On the other hand it is well known the phenomena of Jet-Lag or Jet-Led in airplane trips, being necessary a rest of few hours for solving this situation, during, for instance, over the ocean long trips.

With all this knowledge acquired during the last years, the truth is that we still do not know what role is served by sleep. 


\section{Memory and the problem of automata}

\section{reproduction'}

Our hypothesis is that memory is encoded by the relationships between cells of a network and not by the cells directly. In our approach, when a sequence of symbols has to be stored in memory it is encoded, for instance, in a binary form, and then maintained without any further processing. When it has to be recalled, the signal to recover it gives a start stimulus to the store of the memory which apparently is blank and has no ostensive content. When the signal arises the storage network is effectively blank. But it starts producing the original message, this according with the laws of functioning of the storage network.

We did show that a message of length $\mathrm{n}$ can be stored by a sequence that in the meantime is $2 . \log _{2}(n)$. This has the additional advantaged that messages can be compressed and sent in abbreviated form.

Essentially, the problem of memory is, in this light, given a certain sequence of symbols, to synthetize and automaton that reproduces it when is asked to do it.

Working memory is currently studied under the concepts of immediate memory, work memory, short-term memory and long-term memory.

From a physicalist point of view, memory concerns a purely sequential time, in which present is a single point as in physics.

Nevertheless as Edmund Husserl (1964) has analyzed in the Perception of a Melody, the present experience of the melody I hear is connected to the experience of a passed sound in a threshold memory and the anticipation of future sounds of the melody. Here, present experience is mixed to past and

\footnotetext{
${ }^{9}$ With the collaboration of Paulo Castro, Centre for Philosophy of Science of the University of Lisbon
} 
simultaneously to future. This is much more close to subjective sense of time, whose understanding is crucial for the comprehension of memory processes. A memory of a scene is complex and its memory cannot be reduced to a snapshot. As a matter of fact a complex interaction in a situation has many angles from which it can be seen. It is an invariant under a group of transformations defined from a viewpoint of these different approaches that has to be encoded in memory. Not a single static representation.

During a congress in USA, I asked Professor Hyden about this question, but he did not show sensibility to it, and continued to assert that RNA messenger transmitted the content and stored it. Here begins another question, namely if memory is supported by new structures or how ultimately we can sustain that it maintains during periods of years without degradation. I can see a beautiful teenager and recognize her twenty years later, twenty pounds heavier, and by no means equally beautiful. In a current view of memory, initial conjectures posited that a circulating set of data actively maintained memory.

There are many different types of memory like for instance short-term memory, long-term memory, and work memory. The problem memory poses is how can we explain that seeing one person during a few minutes we can remember it ten years after without any communication during the intermediate time? We will concentrate on this problem. Since the Central Nervous System (CNS) functions with a relevant number of errors, it is not believable that, under these conditions, memory will maintain this integrity without degradation of content.

In our proposal, entirely speculative, we putatively admit that a sequence of digits is stored and maintained, and later recalled in a passive manner. When the recall signal enters an impulse gives a start signal to the storing neural network and this neural structure, starting from zero contents, begins 
producing and reproduces the original sequence. We have to clarify an additional point: how is this storing structure generated? We propose that initially those storing networks are densely connected trough axon and dendrites. The RNA messenger comes, altering the generation of proteins and produces a pruning of this connections. This pruning of dendrites synapses and axons is well-known as cito-architectonics structures of the brain are mentioned which suffer extensively pruning processes during adolescence. If these processes do not take place, apparently subjects appear more prone to suffer schizophrenia. Let us consider the behavior of the following network.

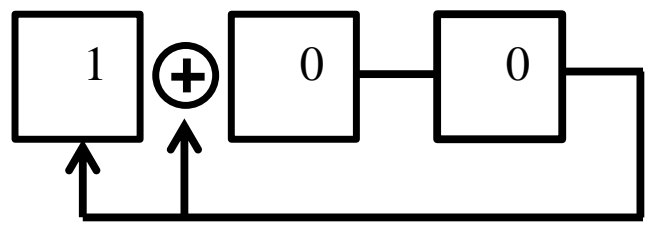

Fig. 3 Shift register feedback circuit with exclusive-or logical operator

We will represent now sequentially the successive states of the network after the initial impulse which is not repeated.

$$
100010001110011111101100
$$

This sequence of states forms a cycle of states of the network with length 7, that is $2^{3}-1=7$, in terms of artificial neurons of the Manuel Blum - McCulloch type. Let me show that with interaction of effects, we can represent exclusiveor. 


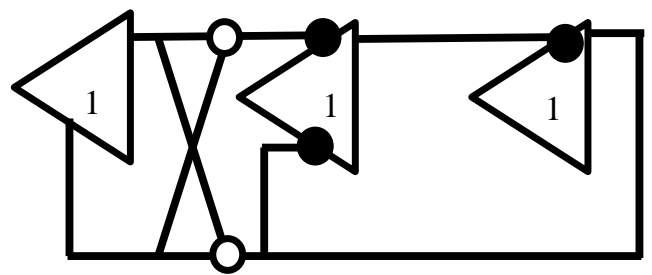

Fig. 4 Circuit in terms of artificial Neurons of the Manuel Blum - McCulloch type, Equivalent to the circuit formed by shift register diagram and an exclusive-or operator

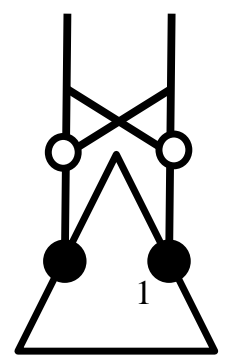

Fig. 5 Exclusive-or

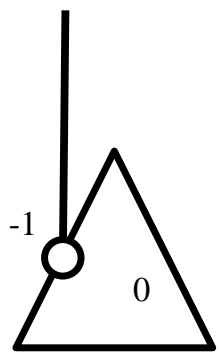

Fig. 6 Negation 


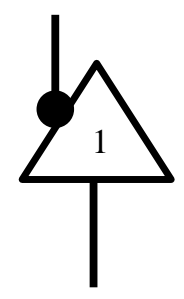

Fig. 7 Delay

In Wittgenstein terms we have the truth tables for the two cases, respectively:

\section{Exclusive-Or}

\begin{tabular}{|lllll|}
\hline $\mathrm{X}$ & 1 & 1 & 0 & 0 \\
$\mathrm{Y}$ & 1 & 0 & 1 & 0 \\
$\mathrm{Z}$ & 0 & 1 & 1 & 0 \\
\hline
\end{tabular}

Negation

\begin{tabular}{|lll|}
\hline $\mathrm{X}$ & 1 & 0 \\
$\mathrm{Y}$ & 0 & 1 \\
\hline
\end{tabular}

Delay

\begin{tabular}{|ll|}
\hline $\mathrm{X}$ & 1 \\
$\mathrm{Y}$ & 1 \\
\hline
\end{tabular}

Fig. 8 Examples of Manuel Blum - McCulloch type neurons

Concluding these breve remarks about memory, we will recall one of the problems in automata studies which concerns John Von Neumann and his problem of given a message of an automaton to find the structure of the 
automaton which produced it. This is just what we did in a heuristic nonrigorously formal way.

The rules are: (a) a sequence has to begin with a 1; (b) an interaction of 1 's is needed if this initial sequence exists; (c) if a 0 appears, the problem can be handled by the introduction of an exclusive-or operator before the neuron which will have state 0 , which is obtained by an incoming feedback which participates in the logical operation, together with the previous input signal; (d) Sequences of 1's and 0's have to be calculated in their length, relatively to feedback re-information in order to avoid interferences; (e) In the case indeterminations occurs:

$$
\begin{aligned}
& S(t) \rightarrow S(t+1) \\
& S(t) \rightarrow U(t+1) \\
& R(t) \neq U(t+1)
\end{aligned}
$$

This indetermination is lifted by the introduction of a new computational element to define conveniently a new logical function.

$$
\begin{aligned}
& x \rightarrow y \\
& x \rightarrow z \\
& z \neq y
\end{aligned}
$$

Now let us discuss rigorously in a mathematical form, more consequences of this algorithm. 


\section{Synthesis and linearization of nonlinear feedback shift registers - basis of a model of memory}

Linear shift registers have been extensively studied since the pioneering work of D. A. Huffman (1955). A general theory was created by Huffman, Golomb, Elspas, Stern, Friedland, Zierler, Hartmannis, Massey, and others. This progress was mainly due to the identification of the operations performed by linear switching circuits with algebraic operations over Galois fields. There was no general theory for nonlinear shift registers, however.

We shall present: (i) a general method of linearization of nonlinear feedback shift registers with or without inputs, which allows us to handle them by the theory of linear switching circuits; (ii) an algorithm to specify a nonlinear net with a minimal number of delays that generates in its autonomous behavior a desired output sequence; (iii) a method of information storage that, for the average of possible cases, gives a logarithmic reduction in space occupancy; (iv) a class of events embedded in a time sequence that extends to the infinite past, and for whose computation any desired degree of reliability can be obtained by using methods that are adequate for definite events having the same length as the readout operation; and (v) some consequences concerning memory and a wide class of learning processes.

\subsection{Method of Linearization}

Our approach extends the Fukunga (1964) method for the autonomous behavior of nonlinear feedback shift registers to the general case of any feedback shift register with any number of inputs through any logical gate.

THEOREM 1: Any feedback shift register with any number of inputs through any logical gate always admits a linearized form by introducing a finite set of new variables. 
Proof: Given a net with input variables $x_{1}, x_{2} \ldots x_{n}$ and variables that define the state of the delay elements $y_{1}, y_{2} \ldots y_{m}$, we can always write the expression that gives the next value of any variable corresponding to a delay element.

$f\left(x_{1}, x_{2}, \ldots, x_{n}, y_{1}, y_{2}, \ldots, y_{m}\right)$, in its normal disjunctive form.

Let the following expression be the normal disjunctive form of this function:

$$
\begin{array}{r}
f\left(x_{1} \ldots, x_{n}, y_{1}, \ldots, y_{m}\right)=a_{0} x_{1} \ldots x_{n} y_{1} \ldots y_{m} \mathrm{Va}_{1} \bar{x}_{1} x_{2} \ldots y_{1} \ldots y_{m} \mathrm{~V} \\
\ldots a_{1} \bar{x}_{1} x_{2} \ldots y_{1} \ldots y_{m}
\end{array}
$$

As the terms in Eq. 1 correspond to mutually exclusive cases, we can substitute $\oplus$ for $V$, and as for any variable $x$,

$$
\bar{x}=x \oplus 1 \text { (modulo } 2)
$$

and

$$
x \oplus x=0 .
$$

By making the corresponding substitutions in (4), we obtain

$$
\begin{aligned}
& f\left(x_{1}, x_{2}, \ldots, x_{n}, y_{1}, y_{2}, \ldots, y_{m}\right)=b_{o} 1 \oplus \\
& \oplus b_{1} x_{1} \oplus \oplus b_{n} x_{n} \oplus b_{n+1} y_{1} \oplus \ldots \oplus a_{n+m} y_{m} \\
& \oplus b_{n+m+1} x_{1} x_{2} \oplus \ldots \oplus b_{n+m+K^{y}} y_{m-1} y_{m} \oplus \\
& \oplus b_{n+L+1} x^{x} 2^{x_{3} \ldots}
\end{aligned}
$$




$$
\oplus b_{r} x_{1} x_{2} \ldots x_{n} y_{1} y_{2} \ldots y_{m \prime}
$$

Where any $b_{i}$ is a constant, either 1 or 0 , and because of (6) no term appears more than once. We note that this is the "exclusive or canonical form", and therefore, in general, for a net with $n$ inputs and $m$ delay elements in order to linearize it we shall have to introduce $2^{n+m}-(n+m)$ variables. Since the state of the delay elements of the circuit can be expressed by an equation of the form of (7), the state of the circuit will be completely defined by $m$ linearized equations.

In many cases this method is a very efficient way of obtaining Boolean functions in a very simplified form.

Example 1: $\quad$ Let $f(x, y, z)=\bar{x} y z V x \bar{y} z V x y \bar{z} V \bar{x} \bar{y} \bar{z}$.

Then $\quad$ by $(1)=\bar{x} y z \oplus x \bar{y} z \oplus x y \bar{z} \oplus \bar{x} \bar{y} \bar{z}$

by (2)

$=(x \oplus 1) y z \oplus x(y \oplus 1) z \oplus x y(z \oplus 1) \oplus(x \oplus 1)(x \oplus 1)(z \oplus 1)$ by (3) $=x \oplus y \oplus z \oplus 1$;

That is, simply the constant 1 added to the sum of the original variables; hence, the only "new" variable introduced is a dummy variable whose value is constrained to be 1 .

This expression can be implemented in the form
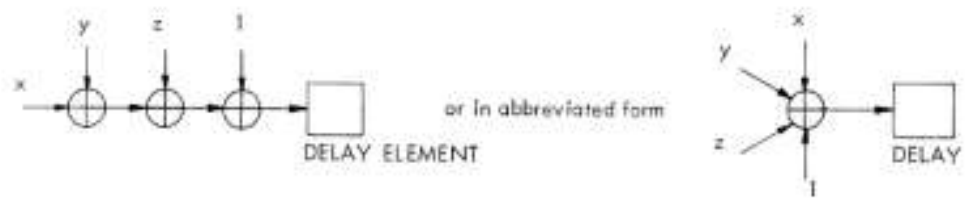
Fig. 2 The function (8) in a spatial representation

An immediate generalization is the extension of the method of linearization to nonlinear algebraic expressions of $n$ variables where the operations are performed over $G F(p), p$ a prime. In that case in order to linearize the expression it will be necessary to introduce, at most, $p^{n}-n$ new variables.

a) Application of the Theory of Linear Switching Circuits to the Linearized Feedback Shift Registers with Operations Defined over $G F(p), p$ a prime

The state of a delay element of a linear switching circuit in any given epoch is determined by a linear combination of a subset of the inputs to the net and of the outputs of a specified subset of the delay elements of the circuit

$$
V=a_{0} x_{1} \oplus \cdots \oplus a_{n} x_{n} \oplus a_{n+1} y_{1} \oplus \cdots \oplus a_{n+m} y_{m}
$$

The terms that are present after linearization and simplification form two classes, A and B.

$\{A\}$ is a class with terms of the linearized form in which no input variable is present.

$\{\mathrm{B}\}$ is a class with terms in which at least one input variable is present.

Let $\vec{S}_{j}$ be a vector whose $\mathrm{n}$ components are the states of the $n$ delay elements of an nth-order shift register in a given epoch, $j$. This vector defines the state of the net in that epoch.

Let $\vec{I}_{j}$ be the state of the u external inputs at the end of epoch $\mathrm{j}$, and $T$ be the n.u matrix that, for every delay element, specifies the subset of delay elements whose state in epoch $j$ determines its state in epoch $j+1$. 
Let $U$ be the $n \times u$ matrix that, for every delay element, specifies the subset of $\vec{I}_{j}$ whose state at the end of epoch $j$ determines its state in epoch $j+1$.

Then the state of a circuit in any given epoch $j+1$ is given by

$$
\vec{S}_{j+1}=T \cdot \vec{S}_{j} \oplus \mathrm{U} \cdot \vec{I}_{j} .
$$

Autonomous behavior is defined as the behavior of a net when its input is constantly kept at 0 . In that case, Eq. 5 becomes

$$
\vec{S}_{j+1}=T \cdot \vec{S}_{j}
$$

By using the definitions of autonomous behavior and of $\{\mathrm{A}\}$ and $\{\mathrm{B}\}$, the only terms that contribute to that behavior will belong to $\{\mathrm{A}\}$.

The nonlinear $n^{\text {th }}$ order net will be embedded in a bigger linear net and the structure of the cycles, transients, and stability states of the nonlinear net will be a subset of the structure of cycles, transients, and stability states corresponding to the linearized net, with the following constraints:

(a) No cycle of the linear net that might correspond to a cycle of the nonlinear net may have a length greater than $2^{n}, n$ being the number of the delay elements in the nonlinear net.

(b) All states that belong to transients that lead to the $00 \ldots 0$ state of the linear net may never correspond to a state of the nonlinear net, when, after linearization, a constant 1 remains in the expression that specifies any of the Boolean functions of the linearized net.

(c) The sum of all possible cycles, transients, and stable states of the linear net corresponding to the nonlinear behavior must add to $2^{n}$. 
(d) The new terms that belong to $\{\mathrm{A}\}$ cannot be supposed to take values incompatible with the values assumed by the initial variables on which they depend.

The structure of cycles to which the preceding constraints apply has been completely handled by Elspas (1959) and Friedland (1959) for the case of operations modulo $p$ ( $p$ any prime) to which all of the preceding arguments may be extended. Elspas obtains that structure from the characteristic polynomial of the matrix that specifies the autonomous behavior.

Consider now the case in which all of the inputs are constantly maintained as 1. It is immediately apparent, for functions over the Galois field (5), that the Boolean expressions that give the state of any of the delays can now be transformed and possibly further simplified by substituting " 1 " in this expression for all input variables and by applying (6) to it.

The behavior of the net under constant input 1 will be equivalent to the autonomous behavior of the net which is obtained when the substitution and simplification are done.

A new type of behavior can be defined that consists in keeping $n-k$ inputs at value 1 , and $k$ inputs at value 0 . The adequate substitution will lead, in general, to a different set of expressions for every different assignment of the inputs that are kept constantly at 1 . All of those cases can be dealt with as if they corresponded to an autonomous behavior of the net that is obtained after substitution of the corresponding input variables by 1 and after simplification.

As all of the results are valid for operations modulo $p$ ( $p$ a prime), any system in which the operations are over a finite set of numbers can be approximated by operations on a Galois field, by mapping from the real numbers that are in one of a set of specified intervals to one element of the field. 
When the source statistics are known, Shannon's (1959) rate-distortion function specifies the number of p-ary digits per second that are required to represent a given information source within some specified fidelity criterion. A common fidelity criterion is the meansquare error.

\subsection{Minimal Nonlinear Net Specification and Synthesis Algorithm}

Let $L$ be a length of an ordered sequence, $S$, of binary digits $s_{L}, \ldots, s_{2}, s_{1}$. Let $S_{n}$ be any subsequence of $S$, of length $n$,

$$
s_{j+n}, \ldots, s_{j+2}, s_{j+1}
$$

We shall now determine the conditions, given $S$, to obtain it as the output of a nonlinear feedback shift register with a minimal number of delay elements, in its autonomous behavior.

THEOREM 2: Given any finite sequence $S$ of binary digits, it is always possible to specify a nonlinear feedback shift register with a minimal number of delay elements that produces that sequence as a cycle of its autonomous behavior by means of the following algorithm.

(0) Set $n=0$. If $s_{1}=s_{2}=\ldots=s_{L}$, go to (3); otherwise, set $n=1$ and go to (4).

(1) Form the L subsequences of length $n S_{n}(j)=s_{j+n}, \ldots, s_{j+2}, s_{j+1}$ $\mathrm{j}=0,1, \ldots, L-1$ (where we define $\left.S_{L+i}=S_{i}\right)$.

(2) For each $i<j$ such that $S_{n}(i)=S_{n}(j)$, compare $s_{j+n+1}$ to $s_{i+n+1}$. If equality always occurs, go to (3); otherwise, increase $n$ by 1 and go to (1).

(3) Stop. The sequence $s_{L}, \ldots, s_{2}, s_{1}$ can be generated from an $n^{\text {th }}$-order nonlinear feedback shift register. 
Every $S_{n}(j)$ can be considered as a state vector of a shift register with digit $S_{j+n+1}$, as the feedback digit that defines the last component of $S_{n}(j+1)$, as well as the desired output that corresponds to $S_{n}(j)$.

It is seen immediately that this interpretation of $S_{n}(j)$ leads to the definition of the successive states, from $\vec{S}_{n}$ to $\vec{S}_{n}(j)$ of a shift register with feedback over the first element that produces $S$ in its autonomous behavior.

For the distinct $S_{n}(j)$ resulting when the algorithm terminates, the output of the Boolean feedback function is determined by the value of $s_{n}(j+1)$. The value of the Boolean feedback function may be arbitrarily chosen for all other states to completely specify the nonlinear feedback shift register. If $2^{n}>L$, there exist $2^{n}-L$ states of the circuit for which the feedback value that corresponds to the more convenient construction can be arbitrarily assigned.

Then, as the behavior of all of the elements of a net is completely specified if all of its states are known, it follows that the circuit that produces $S$ as an output of its autonomous behavior has been completely specified. The procedure further ensures that the circuit has minimal length.

Further steps that lead to an implementation of the net may be outlined as follows. Make a truth table (Magleby, 1963), in which the $\vec{S}_{n}(j)$ appear in the order of their succession in the sequence, and each $\vec{S}_{n}(j)$ is put in correspondence with the last digit at the right of $S^{\mathrm{I} \ldots \mathrm{I}}$. We note that the feedback is necessary only to specify the state of the first element of the circuit. All other elements are merely delay elements. If a linearized form is desired, then the normal disjunctive form for any of the elements is obtained from the truth table, and the procedure of linearization and simplification is applied.

A ratio of compression may be defined for any $S$ as 


$$
R_{c}=\frac{\log _{2} L}{n}
$$

Where $L=$ length of S .

If $2^{n}=L$, then

$$
R_{c}=\frac{\log _{2} L}{\log _{2} L}=1
$$

THEOREM 3: The upper bounds for the median and average lengths of a nonlinear feedback shift register that has a minimal number of delays and can generate in its autonomous behavior a given purely random binary sequence of length $L$ will be

$$
\ell_{m}<2 \log _{2} L
$$

and

$$
\ell_{a v}<3 \log _{2} L+\frac{1}{2}
$$

Proof: (James L. Massey's (1967) proof)

Let $s_{1} s_{2} \ldots s_{\ell}$ be the sequence, where the $s_{i}$ are independent variables, and $P_{r}\left(s_{i}=1\right)=\frac{1}{2}$.

Choose any $i<j$, then

$$
\operatorname{Pr} \overbrace{\left(s_{i} s_{i+1} \cdots s_{i+\ell-2} s_{i+\ell-1}=s_{j} s_{j+1} \cdots s_{j+\ell-2} \bar{s}_{j+\ell-1}\right)}^{\text {event } E_{i j}}=2^{-\ell}
$$


That is, the event that the first $\ell-1$ digits agree, but the last digits differ.

Let

$f\left(s_{1}, s_{2} \ldots s_{L}\right)=$ length of shortest nonlinear FSR that can generate $s_{1}, s_{2} \ldots s_{L}$.

Then

$\operatorname{Pr}(f>\ell)=\operatorname{Pr}$ that $s_{1}, s_{2} \ldots s_{L}$ contains two identical, except for the last digit in which they differ, length $\ell$ subsequences, starting in different positions

$$
\begin{aligned}
& =\operatorname{Pr}\left(U_{j} E_{i j}\right) \leq \sum_{i<j}^{\Sigma} \operatorname{Pr}\left(E_{i j}\right) \\
& =(1+2 \ldots+L-1) 2^{-\ell}=\frac{1}{2}(L-1) L \cdot 2^{-\ell}
\end{aligned}
$$

Let $\ell_{m}=$ median length required: that is,

$$
P\left(f \geq=\ell_{m} \triangleq \frac{1}{2}\right.
$$

But $P\left(f \geq l_{m}\right) \leq \frac{1}{2}(L-1) L 2^{-\ell_{m}}$, by the equation above

$$
\therefore \frac{1}{2} \leq \frac{1}{2}(L-1) L 2^{-\ell_{m}}
$$

or

$$
2^{+\ell_{m}} \leq(L-1) L<L^{2}
$$




$$
\ell l_{m}<2 \log _{2} L
$$

Let $\ell_{\mathrm{av}}=$ average length required; that is,

$$
\ell_{a v} \triangleq \sum_{\ell=0}^{L} \ell \operatorname{Pr}(f=\ell)
$$

But for any integer $\ell^{\prime}$

$$
\begin{gathered}
\ell_{a v} \sum_{\ell=0}^{l^{\prime}} \ell \operatorname{Pr}(f=\ell)+\sum_{\ell=\ell^{\prime}}^{L} \ell \operatorname{Pr}(f=\ell) \\
\ell_{a v}<\ell^{\prime} \operatorname{Pr}\left(f<\ell^{\prime}\right)+L \operatorname{Pr}\left(f=\ell^{\prime}\right) \\
\leq \ell^{\prime}+L \operatorname{Pr}\left(f=\ell^{\prime}\right) .
\end{gathered}
$$

Choosing $\ell^{\prime}=3 \log _{2} L$ yelds

$$
\begin{gathered}
\ell_{a v}<\ell^{\prime} \operatorname{Pr}\left(f<\ell^{\prime}\right)+L \operatorname{Pr}\left(f=\ell^{\prime}\right) \\
\leq \ell^{\prime}+L \operatorname{Pr}\left(f=\ell^{\prime}\right) . \\
\ell_{a v}=3 \log _{2} L+L . L \frac{1}{2} L(L-1) 2^{-\ell^{\prime}} \\
<3 \log _{2} L+\frac{1}{2} L^{3} 2^{-3 \log _{2} L}=3 \log _{2} L+\frac{1}{2} \\
\therefore \ell_{a v}<3 \log _{2} L+\frac{1}{2}
\end{gathered}
$$




\subsection{Method of Informative Storage}

The preceding algorithm gives the possibility of storing a sequence of binary digits not as a stable state but as a pattern of connections and logical relations between delay elements of a shift register.

The basic strategy is this: Rather than designing a neural net to store binary input sequences, one designs a neural net capable of implementing the algorithm of the preceding section and of organizing the minimal length nonlinear feedback shift register. Then when a binary input sequence is processed by the neural net, the resultant nonlinear feedback shift register can be used, upon command, to reproduce the sequence. In other words, a logical structure is stored, rather than the data. We assume that the following conditions are met: (i) The shift register is supposed to be maintained in a 00 ... 0 state in all the epochs between readout modes, by means of a reset process activated in each epoch, the success of which realizes the state $00 \ldots 0$ in the shift register. (ii) This reset process has a probability $P_{1}$ of being applied in any epoch and probability $Q_{1}=1-P_{1}$ of failing to be applied. Furthermore, it is assumed that the reset process acts periodically with period $L$ epochs during the readout mode.

Let $p$ be the probability that the next state of the shift register in the readout mode will be the correct successor for the shift register specified by the fixation algorithm. Any such $n^{\text {th }}$-order feedback shift register produces a sequence of binary digits, which together with the digit that corresponds to the first reset operation should constitute a cycle. The probability that the sequence of states of the shift register contains no erroneous successor state is $P_{s}=p^{L}$, as follows from the definition of $p$ and from the length of the sequence. 


\subsection{Reliability in the Computation of Finite Events Embedded in a Time Sequence That Extends to the Infinite Past}

THEOREM 4: Under the specified conditions (i) and (ii), the probability of occurrence of the desired cycle of length $\mathrm{L}$ following the reset epoch in the readout mode is at least $P_{1} \times p^{L}$. That is, arbitrary reliability in recalling a sequence from the arbitrarily distant past can be obtained by taking measures to ensure the reliability of the operations that occur during $L+1$ epochs.

Proof:

$\operatorname{Pr}[\vec{S}(t)=\overrightarrow{0}]=\operatorname{Pr}[$ reset is applied at time $t]$ $+\operatorname{Pr}$ [reset is not applied at time $t] \mathrm{X} \operatorname{Pr}$ [successor of $\vec{S}(t-1) i s \overrightarrow{0}]$ $\geqslant \operatorname{Pr}[$ reset is applied at time $t]=\mathrm{P}_{1}$.

As $P_{S}=p^{L}$ a a lower bound for the occurrence of $S$ that is completely independent from $t-k$ for $k \geqslant 1$ is given by $P \geqslant P_{1} \times p^{L}$. This is a lower bound and not an exact probability because it ignores the possibility that the correct sequence may be produced even when the re-set operation is not successfully applied.

On the other hand, if we look at the output exclusively, then it may have happened that (i) the reset operation was effective but was followed by any combination of correct states and errors such that the correct output has been preserved, although at least one error in the state of the shift register has occurred during the sequence of length $L+1$. (ii) The reset operation was not effective, but the previous state $\vec{S}(t-1)$ was a predecessor of $\overrightarrow{0}$ and the same succession of conditions as expressed in (i) has happened. (iii) The reset operation was not effective and the previous state $\vec{S}(t-1)$ was not a 
predecessor of $\overrightarrow{0}$, but the same succession of conditions as expressed in (i) or (ii) has happened.

THEOREM 5: When the measures taken to make reliable the re-set operation that immediately precedes the $L$ sequence tend to have absolute reliability without ever attaining it, then, in the limit, the generation of the sequence $S$ will depend on a Markov process of order $L+1$.

Proof: This follows from the fact that the probability of cases (ii) and (iii) will vanish when $P_{1} \rightarrow 1$, since then case (i) always occurs.

\subsection{Consequences Concerning Memory Models and Learning Processes}

The preceding algorithm and the method of section 3 satisfy many of the requirements that can be specified for a formal model of memory when one takes into account current hypotheses about permanent storage in the N. S.

Namely, the contents of memory are not exclusively represented by the actual state of neural elements. The permanent storage of data will be neither as a state vector that shifts cyclically in a reverberatory manner, nor a stable state. The strategy depends on relations between events embodied in neural nets as patterns of connection and modes of operation.

Another feature of the model consists in an active process that periodically drives the state of all the elements to zero. The actual content of the memory is intended to be the zero state, except during recollection and recognition.

From this point of view, the consequences of our model will be that our algorithm or Massey's algorithm for linear shift-register synthesis can be regarded as a model for the process through which structures of the paleocortex, probably with cooperation of the corpora mammillaria and hippocampus, transform a transient input sequence into a permanent structural organization of neural relations. 
For that purpose, a highly reliable set of neurons computing an algorithm of the type proposed here would impose weights in a matrix of neuronal connections capable of all of the possible modes of oscillation in such a way that only the specified mode remains.

The main difference, concerning reliability, is that we have supposed previously that we could specify without an error any desired shift register, and this is not the case with real neurons, for which we have to allow a probability of error in applying the algorithm. Our results suggest the extension of the proposal of shift-register models for the synthesis of macromolecules, which is due to Pattee, in such a way that a scheme similar to the synthesis algorithm may be admitted as an explanation for memory storage by RNA molecules.

No supposition about the kind of plastic changes in the neuronal structure is implied by the present model, although it is clear that it could work either if the relevant changes were in the synapses, or in axonal or dendritic trees, or any combination of these possibilities.

This model suggests, furthermore, a possible mechanism for learning that can occur without needing appreciable repetition of experience as it occurs in certain periods in the lives of young animals. Stated in oversimplified form, in those periods, the occurrence of a certain message in the external input, together with information about the commands that have caused certain actions, leads immediately to a double permanent memory storage, so that when later a certain input is recognized as identical to the past one, it triggers the permanent memory where the sequence of commands for action is stored.

As far as classic and other types of conditioning, as well as trial and error, are concerned, this fixation depends upon the congruence of the results of action over a certain span of time, of congruent associations of external input sequences occurring also in a given span of time. 
At this level of learning, it is reasonable to suppose that, whatever the number of possible actions is, there is a limited number of possible modes of action (Kilmer, McCulloch, \& Blum, 1967), and many trials are necessary, not only to secure an adequate level of congruence but also, probably, to compare them under an abductive process to find the preferable mode (preferable from the point of view of some hierarchical decision function).

Probably the use of the same concept - conditioning - for many different processes, even those that are classified as classical, Type I, Type II, or Operant, is inadequate from the point of view of the function of the nervous system because the levels of the structures upon whose function their occurrence depends may be very different from one case to another.

In a previous note we have suggested a feedback shift register, which is adequate for the command of lower order neuronlike structures or of effectors. The pattern of connections and of logical operations can be such that its output can go through a cycle of states, irrespective of the input. Nevertheless, it has also information about the input and can use it, together with the state of its elements, to form sequences that constitute commands for entraining action. If these acts belong to a certain class that, from the point of view of an observer, tends to certain ends or goals, it is then legitimate to speak of the shift register as producing intentional acts that tend toward certain aims and attain them, taking into account its purpose and the state of the external environment [see (McCulloch, 1965) and (Simões da Fonseca, 1966)].

It is reasonable to postulate that in the brain there is a mapping of the past and the present transactions between the system and the external environment.

This model, in a given situation, would generate sequences of foreseen outcomes of possible actions, taking into account past experience. If they were judged to be of utility from the point of view of a given decision function, the actions would ensue and the commands for them would be stored in an 
enduring form, with the information about the transaction with the external environment.

This kind of performance is usually considered as depending on insight. The same approach could also handle other cognitive processes.

A further remark is that the system is self-organizing, in the sense that an internal structure programs neuronal organization capable of adaptive actions, depending only on experience, the rules of permanent storage, and on a decision about their utility from the point of view of a given criterion of utility.

A model for self-replication can also be immediately derived from our present proposal for permanent memory, and reliable computation in finite lengths of time embedded in the indefinite past.

\section{Neural Nets of Connectivity One That Form Closed Loops Without External Inputs}

Def. 1. The connectivity of a neural net is said to be one whenever any neuron of the net has only one input from another neuron of the net and one output that is the input of only one neuron.

Given a set, $\mathrm{N}$, of neurons $\left\{\begin{array}{llll}n_{1} & n_{2} & \ldots & n_{n}\end{array}\right\}$ with which to form a net that includes all of them and has connectivity one, then (i) any neuron can be connected through its output with any other that does not already have an input; (ii) any neuron can have its input from any other neuron that does not already give its output to another neuron; and (iii) any neuron may perform any one of the four Boolian functions of one variable. 

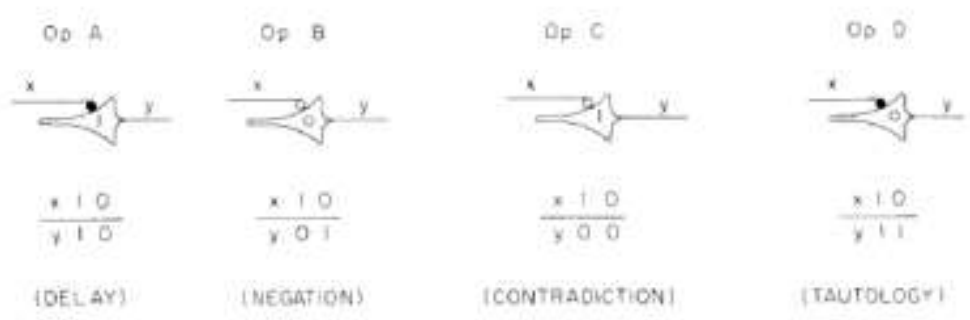

INECATION

Fig. 3 Schematization of Neural Nets of Connectivity

Def. 2. Two nets of neurons, differing only in the number of times that each operation is assigned, and/or, in the order of the assignment, are topologically the same. The pattern of connectivity of any sequence of neurons must have a one-to-one correspondence with the pattern of connectivity of a sequence of neurons in the other. Two nets are independent if they are not connected.

In a former note we reported the establishment of a correspondence between nets of neurons with interaction of afferents, and shift registers. Our present concern is with nets that in some particular cases correspond to linear pure-cycling shift registers, but in others are nonlinear.

Some properties of these nets without inputs and with connectivity one are immediate.

I. The smallest net that can be constructed is formed by two neurons.

II. Any $\mathrm{N}$ neurons (for $\mathrm{N} \geqslant 2$ ) of one output each can be connected to form a net of connectivity one.

III. The smallest number of neurons with which $K$ independent nets can be constructed is $N=2 K$.

IV. Two independent nets of connectivity one, with the same number of neurons can differ only in the number of times each operation is assigned, and/or in the sequence in which these operators are assigned. 
V. Given $N$ neurons, the necessary and sufficient condition for obtaining nets with different topology is that one of these nets has at least two independent subnets, and that in any topology having two or more independent subnets, the number of neurons that form each of at least two of these independent subnets differ from the number of neurons in any of the subnets in which the net of the other topology is partitioned.

VI. The number of possible partitions of a set of $N$ neurons such that the topology corresponding to any of the partitions is different from any other is the number of partitions of $N$ that satisfy the following conditions: (i) they differ in accordance with property $V$; (ii) no independent net has less than two neurons.

VII. In a net of $N$ neurons having at least one neuron that performs functions either $C$ or $D$, no cycle of states is possible.

VIII. If a net of $\mathrm{N}$ neurons is formed exclusively by delay operators, any cycle $N$ length $L=\frac{N}{K}$ ( $K$ being an integer that divides $N$ without remainder) can be obtained.

Proof:

1. If the first state that we assign to the net is either the $11 \ldots 1$ of the $00 \ldots 0$ state, any shift can only produce the same state again. Therefore, a cycle of length 1 is always possible.

2. Let the first state that we assign be the state $10 \ldots 0$ or any other state with no recurrence sequence of ones and zeros whose length divides $N$ without remainder. In the first case all of the digits except one are zero, therefore the number of shifts of the digits along the net necessary to obtain a one in the same position that it had in the first state is $N$. Since at that instant all zeros will also occupy the same positions as they did initially, 
the cycle length $L=N$ is obtained. The same argument applies to any initial state in which there is no recurrence of ones and zeros with length less than $N$.

3. In the assignment of the initial state we can divide the length $N$ in $K$ recurrent sequences of length $\frac{N}{K}$. Then, if we assign to each of these $K$ partitions the recurrent sequences $10 \ldots 0$ with length $\frac{N}{K}$, we note that in any of those partitions the first neuron receives from the $\frac{{ }^{t h}}{K}$ neuron of the preceding sequence the input that it would receive if it belonged to $\frac{N^{t h}}{K}$-order independent net. From (2) it follows that the same cycle of length $\frac{N}{K}$ occurs for every part of the net of length $\frac{N}{K}$. Therefore, the cycle of the total net would be that of any of its parts, that is $\frac{N}{K}$.

IX. In a net of neurons formed exclusively of negation operators we can obtain any cycle length $\mathrm{L}=\frac{N}{K}$ if $L$ is even.

Proof:

1. For $L=1$, the initial state is either $11 \ldots 1$ or $00 \ldots 0$. Since in the next epoch each neuron will negate its input, the state of the net will be, respectively, $00 \ldots 0$ or $11 \ldots 1$. The initial state will be reproduced in the following epoch. Therefore, for $L=1$, the cycle length is $2 X L$; for $L=2$, where the initial state is either $1010 \ldots 10$ or $01 \ldots 01$, each neuron receives as an input the complement of the state in which it is. Since each neuron performs a negation, it remains in the same state.

2. If $L$ is an even number and $L>2$, the same argument as in VIII (3) applies because at the end of the $L^{\text {th }}$ epoch each of the digits 
will have shifted an even number of times. At that time the initial state will be reproduced because the division in $K$ different recurrent subsequences is equivalent to dividing the net in $K$ independent nets with the properties that were shown in VIII. Therefore any cycle length $L$, for $L$ even, $L>2$, and equal to $\frac{N}{K}$, is possible.

3. If $L$ is an odd number, after $L$ units of time, the state of the parts of the net that correspond to the initial recurrent subsequences will be the complement of the initial subsequence, since each digit will be shifted an odd number of times, $L$. Only $L$ times later, will the original subsequence repeat. Therefore, for any $\frac{N}{K}$ that is an odd number, the cycle length will be $2 x L$.

COROLLARY: (a) A net formed exclusively by delay operators always has two possible stable states; (b) A net formed exclusively by negation operators has two stable states if $N$ is even and no stable state if $N$ is odd; and (c) A net that contains at least one operator $C$ or $D$ has one stable state, and any state assigned to it can only be either the stable state or belong to a transient that leads to it.

X. If in a net of $N$ neurons there are $K$ negation operators and $N-K$ delay operators, then for $K>0$ : (i) if $K$ is even, at least a cycle of length 1 and a cycle of length $N$ are possible; (ii) if $K$ is odd, then no stable state may occur and at least a cycle of length $2 . N$ is possible; and (iii) if the distribution of delay and negation operators in the net is such that a given pattern of $m$ neurons with $j$ negations, $j \geqslant 1$, and $m-j$ delay is recurrently repeated, then for every such 
disposition a cycle of length $m$ is possible, if $j$ is even, and of length $2 \times m$, if $j$ is odd.

XI. If $K$ independent nets are constructed of $N$ neurons, the length of the longest possible cycle is $2 \mathrm{Xl.c.m} .\left(1_{a}, 1_{b}, \ldots, 1_{k}\right)$, where $l_{j}$ denotes the number of neurons in any independent net $j$, that is, any of the $K$ partitions of $N$. In this case, the states of all neurons, $N$, are used to define a joint state of the $K$ independent oscillators.

XII. The longest cycle that can be obtained out of the elements of a set $N$ of neurons through any arbitrary partition and/or by assignment of one of the four possible operations to each element of the set, is 2 x l.c.m. $\left(1_{a}, 1_{b}, \ldots, 1_{k}\right)$, as in property IX, with the following conditions: $1_{a}, 1_{b}, \ldots, 1_{k}$, are prime to one another; (ii) $K$ is the highest number of nets that can be obtained from $N$ such that (i) is satisfied; (iii) if more than one partition gives the highest $K$, then the partition that gives the longest cycle is that in which the smallest net of that partition is larger than the smallest in any other partition; and (iv) if in more than one partition, conditions (i), (ii) and (iii) are satisfied, then the partition that gives the longest cycle is that in which the difference between the number of neurons in the greatest and smallest nets of the partition is the least.

\section{Decidability in shift-register theory}

Since the theory of shift registers has been constructed upon the identification of the functions performed by these circuits with algebraic operations over Galois fields, it is of interest to know what the solution is of the problem of decidability for these fields.

Given a field $F$ and a set $S$, of first-order true sentences of $F$, the problem of decision for that field consists in determining whether $S$ is recursive. If $S$ is 
recursive, then $F$ is said to be decidable; otherwise, it is said to be undecidable. For a class, $C$, of fields the problem consists in determining whether the set, $R$, of true sentences in all fields that belong to $C$, is recursive.

Our problems concerning shift registers reduce to finding out whether Galois fields, that is, finite fields, are decidable. The answer [see (Robinson, 1965a) and (Robinson, 1965b)] is that any finite field, $F$, is decidable because any sentence can be reduced to a Boolean combination of sentences of the diagram of $F$, which is finite. 



\section{Consciousness versus Awareness}

The Greek hero of Homer did not behave according to its consciousness states but rather to impulses which were put inside them by the gods. The insight that there was a consciousness as an entity which contributes to voluntary acts appeared in the middle age in an obscure convent of Germany. The nature of consciousness must be differentiated from vigilance. Vigilance oscillates between a high level of attention, somnolence, sleep and coma. On the other hand, in the case of consciousness, normal vigilance is a requirement for it, but to be conscious is to be capable, to render testimony about a situation to which we assist exactly in the same maner as another person, who had assisted to that same situation.

A puzzling question: the bold images of magnetic resonance imaging correspond to a higher or lower contrast provoked by distinct levels of oxygenation of hemoglobin; an organometallic compound. A high oxygenation level is obtained by a higher transport of oxygen by red blood, more saturated in oxygen.

When an area of the brain is activated it's need in oxygen increases, and it's consumption too. On the other hand a more active process may be evaluated as more relevant to adaptation. We have, then, the possibility that bold images reflect, not only a change in oxygen metabolism, but also in the state of consciousness.

We can probably separate the two using tests about the level of awareness and subsequent memory record, to separate psychological from purely metabolic changes without psychological relevance.

In the case this tests implied a higher level of consciousness for active processes of some types, we would be confronted with the puzzling but 
interesting situation of a blood participation in the phenomena of awareness retrieving here Aristotle conception of the soul being a state of blood. 


\section{Herbert Spencer and Hughlings proposal}

\section{for neural central nervous system \\ organization - The problem of positive and negative signs}

Evolutionary processes are represented in the architecture and functioning of the Central Nervous System (CNS) by a hierarchy both morphological and functional.

Morphologically we have to consider by increasing order of differentiation: - rhombencephalon, metencephalon, mesencephalon, diencephalon and thelencephalon.

The most advanced evolutionary structure is the telencephalon.

The concept is that if lesion occurs in some localization there is a regression in the differentiation of functions, globally considered. This is an interpretation of the organization of the central nervous system in terms of the application of evolution theory to the structure and function of the CNS, which is due to the efforts of Herbert Spencer and in medicine, Hughlings Jackson.

In the proposal of Hughlings Jackson (1884) from the lesion of the CNS result two types of consequences: - (a) a lost of the function which was performed by the now lesion structure and (b) liberation of the activity from the control from higher orders centers now destroyed. We have, this way, two types of manifestation, a negative one, which is expressed by loss of function and a positive one which is manifested in liberation from control and that result in a disinhibition and increase of activity. 
In general terms, circumscribed phenomena are seen in neurology and mass phenomena are observed in psychiatry. The Jacksonian point of view has given rise to a layered concept of CNS organization. In successive order we have physiological phenomena; vital and endothermic processes; animic or psychological processes and spiritual processes.

These are the structures of personality as it is conceived by Barahona Fernandes (1966).

To be ill means that this structure is attained globally, what will then generate characteristic symptoms?

This symptoms result from a combination from disturbances in the lower layers with the structures on which normative and ethic processes depend as well as both the individual spirit and the domain of culture. For example in a depression the affective endothermic disturbance is expressed in depressed moods, but also by a lack of pleasure in performing usual activities; diminution of initiative; sense of unpleasantness, and personal de-valuation, and sometimes experiences of guilt and more rarely delusional experiences of ruin with ideas of poverty, hypochondriacally disease which may attain a delusional level, self reference concerning attitudes of the others and sometimes self accusation for some supposed misdeed with negative moral self judgment.

Also, in the case of schizophrenia there is observed a distributed set of disturbances with primarily delusions, that is, ideas that in general are not true, which subjects believe to be true with absolute conviction, not corrigible by logical persuasion. Awareness is clear, some degree of practical incapacity at the level of family, professional occupation, and social environment.

For a diagnosis it is required a minimum of one month of active psychotic process with at least two symptoms of first rank or else one symptom of first 
rank and another symptom which is negative like avolition, autism and poverty of associations.

While in depression there is in general a complete recovery to normality, in the case of schizophrenia one of the criteria for diagnosis is the permanence and no-recovery from negative symptoms. On the contrary, with modern neuroleptic, the recovery from delusional disturbances is obtained after a short while of treatment.

A certain type of disturbance is mania, in which, contrarily to what is observed in depression, mood is euphoric, subjects have a feeling of having their powers enhanced, excess of activity with insomnia, occupational activities in excess and an increased number of pleasant acts which imply an increased risk of harm in economical and in all types of social activity. 



\section{Genetic mutations and auto and self-}

\section{organization - two complementary principles for mutation and natural selection}

Natural evolution from the Darwinian point of view results from mutations that change the behaviour of organisms and if it is negative requires a struggle for survival.

These changes give rise to natural selection of the more able to adapt to environmental circumstances. It results, paradoxically, that small changes with no apparent consequences can be invoked to explain natural selection. Nevertheless, we have to consider that a process of self-organization may amplify the consequences of the mutation and hinder the efforts of the organism to adapt to the new circumstances. For instance, in human psychiatry it may be inherited a diathesis with a propensity for depression, which is not actually expressed ostensibly.

Nevertheless, when conflict does occur, the affected subject can be not able to manage to adapt and a process of remanence, a term due to Tellenbach (1980) makes it remain subjected to the conflict which gives rise to an unfolding of a psychopathological development to depression and melancholia.

This is, in general, the comprehension hypothesis to explain the genesis of processes like neurosis with anxious or obsessive-compulsive character as well as dysthymia with an expression of lighter depressive symptoms.

In alcoholism there may be an inherited propensity to alcohol abuse but the effective occurrence of a disturbance of behaviour and alcohol consumption is linked to life events of conflictive character, exposure to incentives to the 
consumption, psychological conflicts, depressive and anxious stress, life events and social and cultural incentives to alcohol consumption.

Cocaine and amphetamine consumption has become a great and significant social problem as well as a medical and cultural problem. Here the consequences are in psychological terms as severe as high alcohol consumption but in physical health terms, it relatively preserves the mental health of subjects although, in some experiences of these drugs, psychotic phenomena become apparent with health risks and a personality change very difficult to treat, because it is linked to personal beliefs, wishes, as well as social and cultural pressures.

In our community modes of consumption are changing and we observe the appearance of new synthetic drugs with devastating effects in the Central Nervous System (CNS). These drugs are not present and foreseen in legislation, they appear in shops as if they were for other purposes, are cheap, and give rise to severe physical health problems as well as psychiatric manifestations whose consequences can not be foreseen. The main problem with these new drugs is that producers run in such a way that legislation is never completely prepared to classify and allow that control measures can be taken. Smart Shops were recent examples of the exploitation of new examples of economic advantage by stores that practiced a kind of behaviour that can only be compared to the exploitation of prostitution and weapons.

The amount of economic stress that is put on families and welfare system is devastating and poses unsolvable problems to the healthcare system. This has led some political responsible, like in the case of China, to take extremely severe measures, but this is not the case in general as democracy gives to the corrupts the possibility to exercise their action for a long while before they are spotted and forced to behave with a socially acceptable way of life. 


\section{Conclusions}

Painting has no end but beginning. What Leonardo da Vinci says about the representation of reality concerns art as a form of knowledge and his sentence is surely true about many other fields.

If in the sequence of proposals we have been making we consider logon as the particle related to deductive processes and induction and abduction as other two forms of reasoning, emotion and motivation to other areas of mental life, Pavlovian and Skinnerian as particles linked to learning, it seems to us as an useless counterintuitive exercise, as we are used to confessional ways of describing psychic life.

Nevertheless, we have been well succeeded in writing the $p$ wave for many of these phenomena.

Where should we seek the evidence on which we can base the foundation of our descriptions?

At first sight it appears to be lacking, but as a matter of fact, fields of electric potential, on the brain, magnetic fields and in the last years, magnetic resonance due to the spin of protons in the nucleus, have been currently used in medical diagnosis and for research proposals. Event-related potentials have been described as functioning like gates at the entrance of information in the brain, the P50 and N200 or as a contradiction to expectation like the mismatch potential and these phenomena have been relevant to the understanding of changes in the information processing in schizophrenia.

Magnetoencephalographic allows us to examine magnetic fields as the school is transparent to magnetic fields. Differently to what happens to electrical fields of the brain.

Magnetic resonance imagiology, either static or functional, helps us to represent what happens in the brain during relevant behaviors. 
It is this way possible that electrical and magnetic fields may be related to single particle phenomena in the brain. There is puzzling evidence obtained in the brain that points to the fact that current neurophysiological processes would not allow the production of behavior in certain circumstances and nevertheless, it occurs. To explain this impossible occurrence, John Eccles and Karl Popper, a mind body interactionism in which mind would read what is going on at the neurophysiological level but with a remarkable time independent from the neurophysiological process. Sometimes advancing, reading phenomena, not being simultaneous with them, sometimes delaying reading. These phenomena are used by Eccles and Popper to justify a philosophical interactionism as a means to clarify these insolvable biological problems.

José L. Simões da Fonseca and collaborators and the Faculty of Medicine of the University of Lisbon recorded event-related potentials triggered by visual patterns constructed with Campbell spatial sinusoid components. Four classes of visual stimuli where used: (a) vertical sinusoid; (b) horizontal sinusoid; (c) crossed-vertical and -horizontal sinusoid; and (i) white noise, that was obtained according with its mathematical definition using a Poisson distribution of delta waves with equal density for positive and negative waves. This was made in the belief that it is more neutral then a void space. A model of Boolean function was then implemented by helping the subjects to press a button only in the case of crossed sinusoids and of white noise. A model of an equivalence function was built this way and its complement was represented by horizontal and vertical sinusoid. Records were made and their fourier transformed were calculated for each of the four considered situations (two sinusoids, one sinusoid vertical and one sinusoid horizontal and white noise), using multi-varied statistics, stepwise comparisons, a multi-varied analysis was plotted on two axis. Then it was verified that each couple of the 
configurations modelling the Boolean function occupied a similar position in one axis and the other couple on the other. So to speak, conditioned and notconditioned conditions were differentiated along the first axis, and formal differences between each couple of stimuli that was represented by differences in the second axis.

We also observed that in the case of depressive psychopathological disturbances, the separation between couples of classes of stimuli was altered.

Note that here we are not speaking about differences between stimuli, but rather between classes of equivalence of stimuli. It puzzles as if this evidence is not relevant as a field phenomenon to quantum representation of learning.

\section{Final Comment}

Any psychologist will recognize that we have considered problems in very general terms, and, as a matter of fact, in real world interaction between subjects, it is required a much more refined net of qualities to characterize mental processes.

When we analyzed poems of Fernando Pessoa and the letter of Mário de Sá Carneiro, this last with José Carlos Tiago and Dr. Márcia Craveiro, we used another conceptual tool, namely we defined a connotative logic and we substituted sentences of common language by equivalent propositions, written accordingly with this logic of signification. This allows a much more detailed and close interpretation in psychological terms of the mental processes described by poets or common people.

Let us start: we defined connotative logical product as an analog to denotative logical product, but, between, variables defined in intention. In this context if we say "she was very beautiful, but frivolous and not well humored", we can re-write: 
If we add another phrase like "But he loved her"

$$
<\text { but } \otimes \text { he } \otimes \text { loved her }>
$$

And we make the logical product, we join the two propositions.

she was very beautiful, but frivolous and not well humored, but he loved her.

We obtain a complete connotative knowledge of what we think. Suppose that she is no more frivolous, that is this attribute are not true, that does not necessarily means that the sentence is false. If she is no more frivolous, the other still connotatively identify the subject. If he no more loves her, that has a more significant consequence but his change of state doesn't make him a different person.

Continuing the connotative discourse, we can arrive, using what we may call connotative reference to propositions like

$<$ her love was shallow $\otimes$ mainly physical $\otimes$ she got interested in a middle age man she felt was understanding her $\otimes$ left her first love $\otimes$ did fall in love with a second admirer $>$

All are premises for a coming final inference.

Note here that we make the conclusion depend on both connotative references and analytical argument. So, we have here to criteria for truth: (a) analytical truth and (b) connotative referential truth. Considering now another operation, for instance, negation, we may have two possibilities: either the 
negation include all the components of the propositions, or only a part. In this case, an alternative exists in which the original proposition is not completely denied but rather the proposition is transformed into a fuzzy relationship. The same analysis can be made concerning all the alternatives for binary or else anodic relationships and this represents a conceptual departure of conceptual distancing from the purely analytical approach to logics. 



\section{References}

Adrian, E. D. (1922). The relation between the stimulus and the electric response in a single muscle fibre. Arch. Néerl. Physiol., 7, pp. 330332.

Arnold, M. B. (1960). Emotion and personality. New York: Columbia University Press.

Bernard, C. (1974). Lectures on the phenomena common to animals and plants. (C. C. Thomas, Ed.) IL: Trans Hoff HE, Guillemin R, Guillemin L, Springfield.

Bowditch, H. P. (1871). Über die Eigenthümlichkeiten der Reizbarkeit welche die Muskelfasern des Herzens zeigen. Ber. Sachs. Ges., 23, p. 652.

Cajal, S. R. (1934). Les Preuves Objectives de l'Unité Anatomique des Cellules Nerveuses. Trav. Lab . Rech. Biol., Univ. Madrid, 1.

Campbell, F. W., \& Blakemore, C. (1969). On the existence of neurones in the human visual system selectively sensitive to the orientation and size of retinal images. J. Physiol., pp. 203;237-260.

Dirac, P. A. (1981). Principles of Quantum Mechanics. New York: Oxford University Press.

Dubois, D. (1998). Hyperincursive methods for generating fractals in automata related to diffusion and wave equations. International Journal of General Systems, vol. 27, pp. 141-180.

Eccles, J. (1957). The Physiology of Nerve Cells. Baltimore: John Hopkins Press.

Elspas, B. (1959). The Theory of Autonomous Linear Sequential Networks. IRE Transactions, CT-G(6), pp. 45-60. 
Fernandes, H. B. (1966). Um Modelo Teórico da Personalidade em Situação como Ordenador da Psiquiatria Clínica. Jornal do Médico, 61, pp. 625-644.

Fichte, J. G. (2005). The System of Ethics according to the Principles of the Wissenschaftslehre. (D. B. Zöller, Trans.) Oxford: Cambridge University Press.

Fonseca, J. S. (1978). How do normal subjects learn a simple adaptative task: how and why do paranoid schizophrenic patients fail? Arch Psychiatr Nervenkr., 225 (1), 31-53.

Freud, S. (1920). A General Introduction to Psychoanalysis. Boni and Liveright: New York.

Friedland, B. (1959). Linear Modular Sequential Circuits. IRE Trans., CT-6, pp. 61-68.

Fukunaga, K. (1964). A Theory of Non-linear Autonomous Sequential Nets Using Z Transforms. IEEE Trans., EC 13, 301-313.

Fuster, J. M. (1980). The prefrontal cortex. Anatomy, Physiology and Neurophysiology on the frontal lobe. New York: Raven.

Head, H. e. (1920). Studies in Neurology. London: Oxford University Press.

Heidegger, M. (1980). Being and Time. Oxford: Basil Blackwell.

Hodgkin, A. L. (1952). A quantitative description of membrane current and its application to conduction and excitation in nerve. J. Physiol. (London), 117, 500-544.

Huffman, D. A. (1955). The Synthesis of Linear Sequential Coding Networks. In C. Cherry, Information Theory (pp. 77-95). London: Butterworths. Hull, C. (1943). Principles of Behavior.

Hull, C. L. (1952). A Behaviour System. New Haven: Yale U.P.

Husserl, E. (1964). The Phenomenology of internal time-consciousness. The Hague: Nijhoff. 
Jackson, H. (1884). The Croonian Lectures on Evolution and Dissolution of the Nervous System. The British Medical Journal, 1 (1215), pp. 703707.

James, W. (1884). What is an Emotion? Mind, vol. 9, pp. pp. 188-205.

Jouvet, M. a. (1958). Recherches sur l'Activité électrique Cérébrale au Cours du Sommeil. Comptes Rendus des Séances et Mémoires de la Société de Biologie, 152, 1167-1170.

Jung, R. (1958). Excitation, inhibition and coordination of cortical neurons. Experimental cell research, 14, 262-271.

Kandel, E. R., Schwartz, J. H., \& Jessell, T. M. (2013). Principles of Neural Science (Fifth Edition ed.). United States: The McGraw-Hill Companies.

Kierkegaard, S. A. (1989). O Banquete. Guimarães: Guimarães Editores.

Kilmer, W. L., McCulloch, W. S., \& Blum, J. (1967, February 28). Some Mechanisms for a Theory of the Reticular Formation. AF-AFOSR1023-64, Final Scientific Report.

Köhler, W. (1976). The Mentality of Ape. New York London: W. W. Norton. Lange, C. (1922). On Emotions: A Psychophysiological Study. In I. A. Haupt, In The Emotions (I. A. Haupt, Trans., Vol. vol. 1, pp. pp. 33-92). Baltimore: William \& Wilkins.

Lettvin, J. Y., Maturana, H. R., McCulloch, W. S., \& Pitts, W. H. (1968). What the frog's eye tells the frog's brain. In M. B. William C. Corning, The Mind: Biological Approaches to its Functions (pp. 233258). New York: Interscience Publ., a div. of Wiley,.

Lindsley, D. B. (1951). Emotion. In S. S. Stevens, Handbook of Experimental Psychology (pp. pp. 473-516). New York: Wiley.

Lorenz, K., \& Wilson, M. K. (2002). On Aggression. London: Routledge. 
Magleby, K. B. (1963). The Synthesis of Nonlinear Feedback Shift Registers. (S. E. Laboratory, Ed.) Technical Report, No. 6207-1.

Magoun, H. W. (1952). An Ascending Reticular System in the Brain Stem. Ama Archives of Neurology and Psychiatry, 67 (2), pp. pp. 145-154.

Massey, J. L. (1967). Shift-Register Synthesis and Applications. Quarterly Progress Report, 85, pp. 239-240.

McCulloch, W. S. (1965). Embodiments of Mind. Cambridge (Mass.): The M.I T. Press.

McDougall, W. (1913). An Introduction to Social Psychology. London: Methuen and Co. .

Miledi, R., \& Slater, C. (1966). The action of calcium on neuronal synapses in the squid. J Physiol, 184, pp. 473- 498.

Morris, D. (1967). The Naked Ape: A Zoologist's Study of the Human Animal . New York: McGraw-Hill.

Morrison, R. S., \& Dempsey, E. W. (1943). The Electrical Activity of a Thalamo-Cortical Relay System. American Journal of Physiology, pp. pp. 283-296.

Parnas, J. a. (2011). The structure of self consciousness in schizophrenia. In S.

G. (Ed.), The Oxford Handbook of the Self (pp. pp. 521-546). Oxford: Oxford University Press.

Pauli, W. (1925). Über den Zusammenhang des Abschlusses der Elektronengruppen im Atom mit der Komplexstruktur der Spektren. Z. Phys., 31, pp. pp 765-783.

Pessoa, F. (. (1946). Odes de Ricardo Reis. Lisboa: Ática.

Pierce, C. S. (1931-35). Collected Papers of Charles Sanders Peirce (Vols. Vols. I-VI). (C. Hartshorne, \& P. Weiss, Eds.) Cambridge, Massachussets: Harvard University Press. 
Robinson, J. (1965a). Introduction to Model Theory and to Metamathematics of Algebra. Amsterdam: North-Holland Publishing Company.

Robinson, J. (1965b). The Decision Problem for Fields. In J. W. Addison, L. Henkin, \& A. Tarski, The Theory of Models. North-Holland Publishing Company.

Saussure, F. (1959). Course in General Linguistics. New York: Philosophical Library.

Schachter, S., \& Singer, J. (1962). Cognitive, Social, and Physiological Determinants of Emotional State. Psychological Review 69, 379399.

Shagass, C. (1972). Evoked Brain Potentials in Psychiatry. New York: Plenum Press.

Shannon, C. E. (1959). Coding Theorems for a Discrete Source with a Fidelity Criterion. IRE National Convention Record, Vol. 7, Part 4, pp. 142163.

Shannon, C. E., \& Weaver, W. (1949). The Mathematical Theory of Communication. Urbana: University of Illinois Press.

Simões da Fonseca, J. L. (1966). Neuronal Models. Lisbon: Centro de Estudos Egas Moniz.

Strawson , P. F. (1952). An Introduction to Logical Theory. London: Methuen.

Tellenbach, H. (1980). Melancholy. History of the Problem, Endogeneity, Typology, Pathogenesis, Clinical Considerations. Pittsburgh: Duquesne University Press.

Tinbergen, N. (1951). The Study of Instinct. New York: Oxford Univ. Press. Zadeh. (1965). Fuzzy sets and systems. Proc. Symp. on System Theory, 29-39. Zahavi, D. (2005). Subjectivity and Selfhood: Investigating the First-Person Perspective. Cambridge (Mass.): MIT. 



\section{Acknowledgements}

The author thanks Professors José N. Croca, António A. M. Cardoso and Dr. Paulo Castro for their mathematical revision of the text.

Professor Isabel Fonseca and José Carlos Tiago de Oliveira revised the English text, as well as Dr. Emilia Gala.

The author also wants to express his gratitude to Professor Olga Pombo, who accepted our collaboration in the Centre for Philosophy of Sciences of the University of Lisbon, not witstanding being only a Physician.

To Professor José N. Croca, we thank for his inspiring leadership in the Research Area Philosophy of Quantum Physics: Nonlinearity and Eurhythmy, of the Centre for Philosophy of Sciences of the University of Lisbon.

Specially the author thanks Márcia Craveiro who has been his eyes and hands during the writing and edition of this book. Our gratitude for her professional competence never will be enough. 